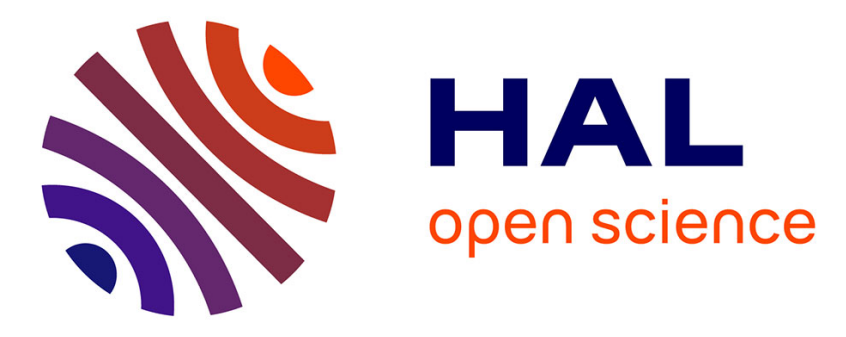

\title{
Laponite/poly(2-methyl-2-oxazoline) hydrogels: Interplay between local structure and rheological behaviour
}

Clémence Le Coeur, Cédric Lorthioir, Artem Feoktystov, Baohu Wu, Gisèle Volet, Catherine Amiel

\section{To cite this version:}

Clémence Le Coeur, Cédric Lorthioir, Artem Feoktystov, Baohu Wu, Gisèle Volet, et al.. Laponite/poly(2-methyl-2-oxazoline) hydrogels: Interplay between local structure and rheological behaviour. Journal of Colloid and Interface Science, 2021, 582 (Part A), pp.149-158. 10.1016/j.jcis.2020.07.068 . hal-02925280

\section{HAL Id: hal-02925280 \\ https: / hal.sorbonne-universite.fr/hal-02925280}

Submitted on 28 Aug 2020

HAL is a multi-disciplinary open access archive for the deposit and dissemination of scientific research documents, whether they are published or not. The documents may come from teaching and research institutions in France or abroad, or from public or private research centers.
L'archive ouverte pluridisciplinaire HAL, est destinée au dépôt et à la diffusion de documents scientifiques de niveau recherche, publiés ou non, émanant des établissements d'enseignement et de recherche français ou étrangers, des laboratoires publics ou privés. 


\section{Laponite/poly(2-methyl-2-oxazoline) hydrogels: interplay}

\section{between local structure and rheological behaviour}

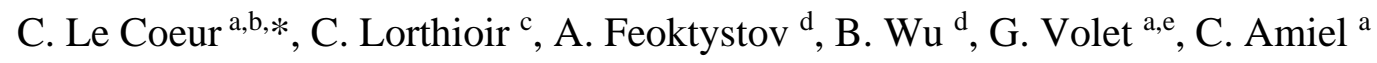

a Université Paris Est Creteil, CNRS, Institut Chimie et Matériaux Paris Est, 2 rue Henri Dunant, 94320 Thiais, France

b Laboratoire Léon Brillouin, CEA-CNRS (UMR-12), CEA Saclay, Université Paris-Saclay, 91191 Gif-sur-Yvette Cedex, France

c Sorbonne Université, CNRS, Collège de France, Laboratoire de Chimie de la Matière Condensée de Paris, 4 Place Jussieu, 75005 Paris, France

d Forschungszentrum Jülich GmbH, Jülich Centre for Neutron Science JCNS at Heinz Maier-Leibnitz Zentrum MLZ, Lichtenbergstraße 1, 85748 Garching, Germany

e Université d'Evry Val d'Essonne, Rue du Père Jarlan, 91025 Evry Cedex, France

\section{* Corresponding author :}

Clémence Le Coeur, E-mail : lecoeur@icmpe.cnrs.fr, Tel. +33.(0)1.49.78.12.86,

Fax: +33.(0)1.49.78.13.08.

E-mail addresses : $\quad \underline{\text { lecoeur@icmpe.cnrs.fr }}$ (C. Le Cœur), cedric.lorthioir@upmc.fr (C. Lorthioir), a.feoktystov@fzjuelich.de (A. Feoktystov), ba.wu@fz-juelich.de (B. Wu), volet@icmpe.cnrs.fr (G. Volet), amiel@icmpe.cnrs.fr (C. Amiel). 


\section{Abstract}

\section{Hypothesis}

Dispersions of Laponite in water may form gels, the rheological properties of which being possibly tuned by the addition of polymer chains. Laponite-based hydrogels with poly(ethylene oxide) (PEO) were the most widely investigated systems and the PEO chains were then found to reduce the elastic modulus.

\section{Experiments}

Here, hydrogels based on Laponite and poly(2-methyl-2-oxazoline) (POXA) were considered. The adsorption behavior and the local structures within these nanocomposite gels were investigated by small-angle neutron scattering and NMR. The same materials were macroscopically characterized using rheology.

\section{Findings}

An original evolution of the storage modulus $\mathrm{G}^{\prime}$ with the POXA concentration is evidenced compared to Laponite/PEO hydrogels. At low POXA concentrations, a continuous reduction of $\mathrm{G}^{\prime}$ is observed upon increasing the polymer content, as with PEO, due to the screening of electrostatic interactions between the clay platelets. However, above a critical value of the POXA concentration, $G^{\prime}$ increases with the polymer content. This difference with PEO-based hydrogels is correlated to the stronger affinity of POXA chains for the clay surfaces, which results in the reduction of the inhomogeneities for the Laponite disks within the gels. Steric repulsions would then counterbalance the effect of electrostatic repulsions and lead to the strengthening of the POXA-based hydrogels.

Keywords: poly(2-methyl-2-oxazoline); clays; hydrogels; nanocomposites; adsorption; SANS 


\section{Introduction}

The addition of nanoparticles in polymer solutions, gels or polymers in the molten state has been widely used to tune the rheological/mechanical properties of these materials. As such, polymer/nanoparticle complex systems have attracted growing interests over the past decades and have led to very different applications in various fields such as paints, cosmetics, therapeutics or tissue engineering [1-3]. In a general way, for such organic/inorganic hybrid materials or nanocomposites, the adsorption of polymer chains on the surface of nanoparticles plays a key role on their rheological/mechanical behaviour [4].

More recently, the incorporation of inorganic nano-objects into polymer-based hydrogels has been developed ([5-8] for instance). Again, for this class of materials, the adsorption of polymer chains (poly $(N$-isopropylacrylamide) [8] or poly(dimethylacrylamide) [9-11]) onto inorganic particles (Laponite platelets in Haraguchi's work [8,9] and silica nanoparticles in Hourdet's work [10,11]) was found to be of primary relevance to control the reinforcement of the plateau modulus. Besides, these nano-objects also affect the hydrogel properties under large deformations: the elongation at break could be increased up to $1600 \%$, a value which is much higher than the one obtained for the unfilled hydrogels $[9,12,13]$. In situ polymerization of $N, N$ dimethylacrylamide performed within a Laponite dispersion in water led to hydrogels with excellent mechanical properties. Indeed, one of the first work by Haraguchi in this field [8] reports a Young modulus of $25 \mathrm{kPa}$ and an elongation at break of $1200 \%$ for a hydrogel with $8 \%$ of Laponite. These hydrogels may be viewed as polymer networks in which the inorganic nanoparticles would act as physical cross-links and in this respect, the complex interactions between the polymer chains and the inorganic particles are 
particularly important to control the dispersion of the filler nanoparticles. In this context, phyllosilicates with a large specific surface area such as Laponite, Hectorite or Montmorillonite have been used as models of inorganic nanoparticles which may develop significant interactions with hydrophilic polymer chains [14-16]. Besides, Laponite is a synthetic clay composed of individual platelets with a well-defined shape: these latter indeed display a disk-like shape with a diameter of $30 \mathrm{~nm}$ and a thickness of $1 \mathrm{~nm}$ [17-20]. From an applicative point of view, Laponite is used in many different fields such as drug delivery [21-23], tissue engineering, cosmetics, or buildings or even for fuels , where it could be used as a thixotropic agent [24]. Hydrogels composed of a nanoparticle network, without any polymer chains, have also been quite extensively studied, in particular Laponite-based hydrogels. In aqueous media, the faces of the Laponite platelets are negatively charged whereas their edges are positively charged. Their dispersions are basic, with a $\mathrm{pH}$ of around 10. The phase diagram of Laponite in water is complex [4,25-27]. At a concentration between 1 and $3 \mathrm{wt} \%$ in water and at low ionic strength, Laponite dispersions display an isotropic gel structure [4,25,28,29]. The detailed description of the platelets' organization within such hydrogels is still a matter of debate. It is commonly admitted that at low ionic strength, the gel structure results from repulsive interactions between the charged clay disks.

These nanoparticle-based hydrogels offer significant interfacial regions, which can be modified by the adsorption of polymer chains. In the literature, numerous nanocomposite hydrogels based on a hydrophilic polymer and Laponite have been studied [6,14-16,30-35]. In particular, the addition of poly(ethylene oxide) (PEO) $[6,14-16,30-32,36,37]$ during the preparation of the Laponite gel has been deeply investigated. With a biocompatible polymer, such composite hydrogels can find application in the field of drug delivery for instance $[38,39]$. In these works, several 
experimental approaches such as small-angle neutron or X-ray scattering (SANS/SAXS) [5,14-16,40], dynamic light scattering (DLS) [41-43], nuclear magnetic resonance (NMR) [37] and rheometry [32,34,36,44] have been used. Both gelation kinetics and rheological behaviour of such nanocomposite hydrogels were found to depend on the adsorption of the polymer chains on the clay surfaces. Along this line, several studies aiming at describing the influence of the PEO molecular weight were reported in the literature. For high PEO molecular weights, hydrogels exhibit the so-called "shake gel" behaviour $[6,15,32,34,45,46]$, meaning that these systems are fluid at rest but display a significant shear thickening at sufficiently high shear rate. The critical value of the shear rate depends on various parameters, among which the PEO concentration. The gel formation is attributed to the adsorption of long PEO chains on the Laponite disks: due to their high degree of polymerization, these chains can bridge clay nanoparticles, thus resulting in a polymeric network under shear. In contrast, low molecular weight PEO chains slow down the gelation of Laponite because of the steric hindrance and the screening of the clay charges $[42,47]$. The critical molecular weight between both regimes corresponds to the one for which the chain radius of gyration matches the average distance between clay platelets. On a local scale, studies on PEO/Laponite systems have provided an understanding of the adsorption of the polymer to the platelet surfaces $([15,19,41]$. These studies have demonstrated that the chains adsorbed flat on the platelets faces and also on its edges. The thickness of the adsorbed polymer layer on the large sides hardly depends on the mass of the polymer, whereas on the edges, the adsorbed thickness seems to vary according to the mass of the polymer (edge thickness $\sim \mathrm{M}_{\mathrm{w}}{ }^{0.13}$ ) [41]. For low molecular weight PEO/Laponite systems, previous dynamical studies have shown that part of the PEO repeat units are adsorbed on the Laponite disks whereas the remaining ones are involved in loops and tails $[37,48]$. During the gelation, 
this latter fraction gets weaker, due to the adsorption of repeat units from loops or tails on neighbouring clay layers.

In the present work, our aim is to elaborate nanocomposite hydrogels based on another hydrophilic polymer, poly(2-methyl-2-oxazoline) (POXA). POXA is quite often considered in the biological context as an alternative to PEO since it is a hydrophilic, biocompatible and bio-inert polymer [49]. POXA has indeed been reported to display an interesting resistance against protein adsorption, making it a promising candidate for the design of nanocomposite hydrogels for tissue engineering. POXA and the complete poly(2-alkyl-2-oxazoline) series enable larger synthetic versatility and show stronger resistance to degradation in a biological environment than PEO [50]. This contribution evidences that POXA displays a different adsorption behaviour on Laponite than the one reported for PEO. The comparison between POXA/Laponite and PEO/Laponite hydrogels enables to highlight the strong correlation between polymer/surface interactions and the rheological properties of such materials. Interestingly, an unprecedented evolution of the storage modulus $G^{\prime}$ with the POXA concentration is detected, compared to the results obtained for hydrogels prepared with a PEO homopolymer characterized by the same degree of polymerization as POXA. Indeed, for low POXA / PEO concentrations, a continuous decrease of G' with the polymer content occurs for both POXA- and PEO-based hydrogels. However, in the case of POXA, the opposite trend, i.e. an increase of $\mathrm{G}^{\prime}$ with the POXA content, is clearly observed above a critical polymer concentration. Such a feature is not observed in the case of PEO. This unprecedented result is rationalized owing to a detailed analysis of the POXA adsorption at the clay surfaces and the analysis of the local structure of these hydrogels by means 
of SANS and NMR experiments. The adsorption of POXA on Laponite disks is investigated, at the molecular length scale, by ${ }^{1} \mathrm{H}$ NMR. The POXA chain conformation as well as the organization of the clay layers within the hydrogels is studied by SANS using the contrast variation technique. The structural information of these hydrogels is then correlated to their rheological behaviour and compared to the ones obtained with PEO of similar molecular weight.

\section{Materials and Methods}

\subsection{Materials}

Poly(2-methyl-2-oxazoline), denoted as POXA in the following, was previously synthetized according to the protocol described in details in [51]. Its number average molecular weight, $M_{\mathrm{n}}$, amounts to $3.4 \times 10^{3} \mathrm{~g} \cdot \mathrm{mol}^{-1}$, with a polydispersity index $\mathrm{M}_{\mathrm{w}} / \mathrm{M}_{\mathrm{n}}$ of 1.1. Poly(ethylene oxide) (PEO) with a molecular weight $\mathrm{M}_{\mathrm{n}}=1915 \mathrm{~g} \cdot \mathrm{mol}^{-1}$ and with a polydispersity index $\mathrm{M}_{\mathrm{w}} / \mathrm{M}_{\mathrm{n}}$ of 1.03 has been purchased from Sigma Aldrich (BioUltra).

Laponite $\mathrm{RD}, \mathrm{Na}^{+0.7}\left[\left(\mathrm{Si}_{8} \mathrm{Mg}_{5.5} \mathrm{Li}_{0.3}\right) \mathrm{O}_{20}(\mathrm{OH})_{4}\right]^{0.7-}$, was a kind gift from Rockwood Additives Ltd., United Kingdom. Deionized $\mathrm{H}_{2} \mathrm{O}$ (MilliQ) was used throughout this study, $\mathrm{D}_{2} \mathrm{O}$ (99.98\% isotopic purity) was purchased from Eurisotop, France and $\mathrm{NaCl}$, from Sigma Aldrich, USA.

\subsection{Sample preparation}

A dispersion of Laponite at $3.0 \mathrm{wt} \%$ in water was obtained by stirring at $500 \mathrm{rpm}$, during $1 \mathrm{~h}$, a volume of $5 \mathrm{~mL}$ in a vial of $10 \mathrm{~mm}$ diameter. This concentration was chosen to form isotropic gels and to remain always in the same part of the Laponite phase 
diagram, for all the hydrogels investigated in this work [4]. Variations of the conditions used during this step may lead to different extents of exfoliation of the Laponite disks and then, to a lack of reproducibility. Therefore, in this work, the very same conditions were used for the preparation of all the samples. This dispersion was consecutively mixed with both a solution of POXA at 80 g.L.-1 in water and an aqueous solution of $\mathrm{NaCl}$ at $100 \mathrm{mM}$. The resulting solution was then stirred during $3 \mathrm{~min}$ at $700 \mathrm{rpm}$. The added volumes resulted in solutions with a concentration of $\mathrm{NaCl}$ fixed to $5 \mathrm{mM}$, a concentration of Laponite ( $c_{\text {lap }}$ ) equal to $1.5,2.0$ or $2.5 \mathrm{wt} \%$ and a concentration of POXA $\left(c_{\mathrm{p}}\right)$ ranging from 0.21 to $1.89 \mathrm{wt} \%$.

\section{$2.3 N M R$}

${ }^{1} \mathrm{H}$ solution-state NMR measurements were performed on a Bruker Avance II spectrometer $\left({ }^{1} \mathrm{H}\right.$ Larmor frequency of $\left.400.2 \mathrm{MHz}\right)$ equipped with a BBFO $5 \mathrm{~mm}$ probe. Single-pulse experiments were carried out using a $90^{\circ}\left({ }^{1} \mathrm{H}\right)$ pulse length of $7.5 \mu$ s and a recycle delay of $5 \mathrm{~s}$, set according to the longest $\mathrm{T}_{1}\left({ }^{1} \mathrm{H}\right)$ relaxation time. All the samples were analysed in neat $\mathrm{D}_{2} \mathrm{O}$.

\subsection{Rheometry}

Rheological measurements were carried out at $25^{\circ} \mathrm{C}$ on a DHR2 rheometer (TA Instruments) using a cone-plate geometry with a diameter of $60 \mathrm{~mm}$ and a cone angle of $1^{\circ}$. All the data were acquired after a fixed time following sample preparation ( 1 day, 2 days, 5 days and 11 days). Oscillatory stress sweeps were carried out at a frequency of $1 \mathrm{~Hz}$ and with a shear strain amplitude $\gamma$ of $1 \%$. Such a deformation amplitude was found to lie within the linear viscoelastic regime, for all the hydrogels investigated. 


\subsection{Small-Angle Neutron Scattering (SANS)}

SANS measurements were performed on the PACE spectrometer at Laboratoire Léon Brillouin and on KWS2 operated by Jülich Center for Neutron Science at Heinz Maier-Leibnitz Zentrum in Garching (Germany) [52]. Three configurations, implying two sample-to-detector distances, were systematically used in order to cover a q-range extending from $2.5 \times 10^{-3}$ to $0.3 \AA^{-1}$. Samples were measured with Hellma cells characterized by a small path length $\left(1 \mathrm{~mm}\right.$ or $2 \mathrm{~mm}$, depending on the $\mathrm{D}_{2} \mathrm{O}$ content). Spectra were corrected for incoherent scattering, cell, background and solvent.

In an aqueous binary mixture of Laponite nanoparticles and polymer chains, the scattered intensity is given by:

$$
\begin{array}{r}
I(q)=\left(\rho_{\text {lap }}-\rho_{s}\right)^{2} \cdot I_{\text {lap-lap }}(q)+\left(\rho_{p}-\rho_{s}\right)^{2} \cdot I_{p-p}(q)+2\left(\rho_{\text {lap }}-\rho_{s}\right)\left(\rho_{p}-\rho_{s}\right) \cdot \\
I_{p-l a p}(q)
\end{array}
$$

where $I_{\text {lap-lap }}$ and $I_{\mathrm{p}-\mathrm{p}}$ correspond to the scattering intensity from Laponite platelets and polymer chains, respectively, while $I_{\mathrm{p}-\text { lap }}$ is the interference term due to the correlations between both components. $\rho_{s}, \rho_{p}$ and $\rho_{\text {lap }}$ denote the coherent scattering length density of the solvent, the polymer chains and the Laponite disks. Due the intrinsic difference between the coherent scattering length density for Laponite and the one for the polymer $\left(\rho_{\text {lap }}=3.91 \times 10^{10} \mathrm{~cm}^{-2}, \rho_{\mathrm{p}}=1.40 \times 10^{10} \mathrm{~cm}^{-2}\right)$, the scattering length density of the solvent $\rho_{\text {solv }}=\left(x \mathrm{D}_{2} \mathrm{O}+(1-x) \mathrm{H}_{2} \mathrm{O}\right)$ may be tuned to $\rho_{\text {lap }}$ or, alternatively, to $\rho_{\mathrm{p}}$. Such matching conditions are fulfilled for $x=0.68$ and $\mathrm{x}=0.25$, respectively, while keeping a significant contrast between the solvent and the scattering objects (polymer chains or clay platelets, respectively). When the contribution from the Laponite (POXA) particles is masked, 
only the contribution from POXA (Laponite) appears as a wave-vector-dependent intensity in the spectra, whatever the Laponite (POXA) concentration.

In such a case, Eq. (1) can be simplified into the following expression:

$$
I(\mathrm{q})=\frac{c(\Delta \rho)^{2} v_{S}^{2}}{N_{A}} \cdot M_{a} \cdot P(\mathrm{q}) \cdot S(\mathrm{q})
$$

$\Delta \rho$ is the neutron scattering length density contrast between the solvent and the scattering object; $N_{A}$ is the Avogadro number; $v_{s}, \mathrm{c}$ and $M_{\mathrm{a}}$ are the specific volume, the concentration and the apparent mass of the scatterer. For POXA, we have used $v_{s}=0.87$ $\mathrm{cm}^{3} \cdot \mathrm{g}^{-1}[53] . P(\mathrm{q})$ and $S(\mathrm{q})$ are, respectively, the form factor and the structure factor of the scatterer. In dilute solutions, one may assume that no interaction occurs between scattering objects, so that $S(\mathrm{q}) \approx 1$. The form factor, $P(\mathrm{q})$, will depend on the type of scatterers observed. For the polymer chains, the Debye form factor is used:

$$
P(q)=\frac{2}{x^{2}} \cdot\left(x-1+e^{-x}\right) \text { with } \mathrm{x}=\mathrm{q} \cdot R_{\mathrm{g}}
$$

from which the radius of gyration, $R_{\mathrm{g}}$, may be derived. For Laponite platelets, the expression of the form factor for a discoidal shape is considered:

$$
P(q)=\int_{0}^{\pi / 2} \sin \theta d \theta V_{p}^{2} \Delta \rho^{2}\left[\frac{\sin \left(q \cdot h \cdot \frac{\cos (\theta)}{2}\right)}{q \cdot h \cdot \cos (\theta) / 2} \cdot \frac{2 J_{1}\left[q \cdot R_{p} \cdot \sin (\theta)\right]}{q \cdot \sin (\theta)}\right]
$$

In this formula, $R_{p}, h$ and $V_{p}$ are the radius, the thickness and the volume of the disk respectively $\left(V_{p}=\pi \cdot R_{p}^{2} \cdot h\right) . J_{1}$ denotes the first-order Bessel function. Fits have been performed with the help of Sas View software [54].

\section{Results and Discussion}

\subsection{Adsorption of POXA on Laponite}


In previous reports on Laponite/PEO hydrogels, the adsorption of PEO chains on the clay platelets has been investigated by SANS or NMR. Cosgrove and coworkers [16] performed NMR and SANS measurements to quantify the adsorption of PEO at the clay surfaces and to determine the plateau value of the adsorption isotherm. Their NMR approach is based on the analysis of the solvent relaxation, which relies on the difference in the ${ }^{1} \mathrm{H}$ transverse relaxation behaviour for water in the bulk and for the solvent molecules at the interfaces. In another work [37], the combination of solution-state and solid-state NMR was used to investigate the PEO segmental mobility and conformations at the clay interfaces. The PEO chain segments close to the Laponite disks were not frozen on the tens of microseconds time scale but were found to display a reduced segmental mobility. In contrast, the PEO chain segments located further from the interfaces comparatively exhibit a much higher level of segmental mobility and their proportion was found to be close to one third of the total amount of PEO units, for a PEO molecular weight $M_{\mathrm{w}}$ of $2000 \mathrm{~g} \cdot \mathrm{mol}^{-1}$.

In the present study, ${ }^{1} \mathrm{H}$ solution-state NMR measurements have been carried out with a solution of POXA in heavy water and POXA chains within a Laponite hydrogel $\left(c_{l a p}=2 \mathrm{wt} \%\right)$, at the same polymer concentration $\left(c_{p}=0.66 \mathrm{wt} \%\right)$. As can be seen in Fig. 1b (upper spectrum), the ${ }^{1} \mathrm{H}$ NMR spectrum of POXA in water displays two broad signals which can be assigned to the methyl protons (at $\delta=1.5 \mathrm{ppm}$ ) and $\mathrm{CH}_{2}$ protons (at $\delta=3.4 \mathrm{ppm}$ ). In the presence of $2 \mathrm{wt} \%$ of Laponite, both peaks related to the POXA units become so broad that they cannot be detected any longer, though the ${ }^{1} \mathrm{H}$ NMR spectrum was recorded a few minutes after mixing (Supplementary Material, Fig. S1). Therefore, in the hydrogel, the segmental motions of the POXA chains are frozen over the characteristic time scale of the experiment, a few tens of microseconds in the present case. Such a slowing-down does not origin from any reorganization of the Laponite 
layers, as observed in reference [19], which is indeed expected to occur over a longer time scale under these experimental conditions. This result indicates that POXA chain segments are fully adsorbed on the clay surfaces and adopt train conformations. In the case of PEO with a similar degree of polymerization and in interaction with silica surfaces, around $45 \%$ of PEO segments were still mobile a few minutes after mixing [55]. One may deduce that POXA has a higher affinity for Laponite than PEO and that the POXA chains display flat conformations at the clay surfaces due to its chemical

(a)

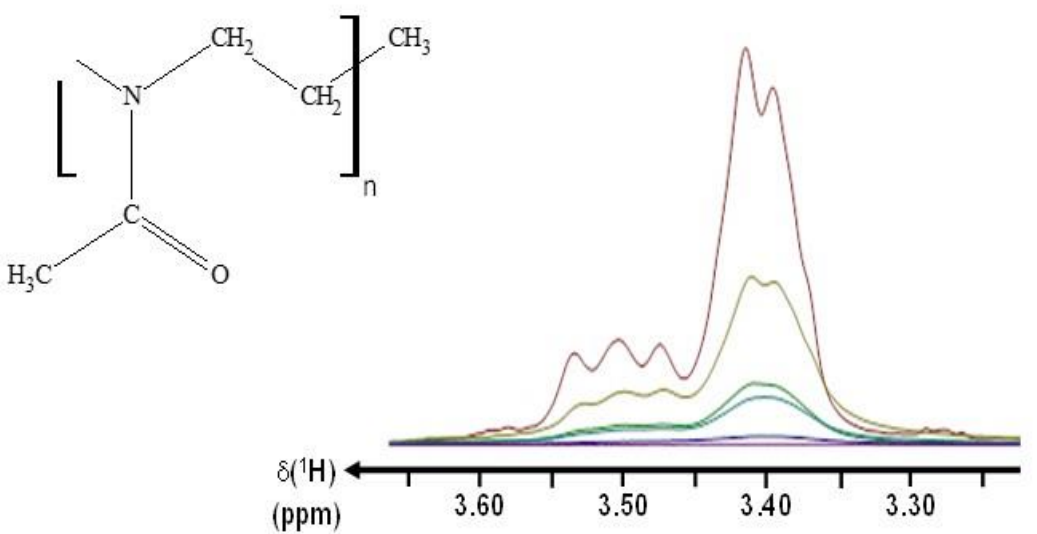

(b)

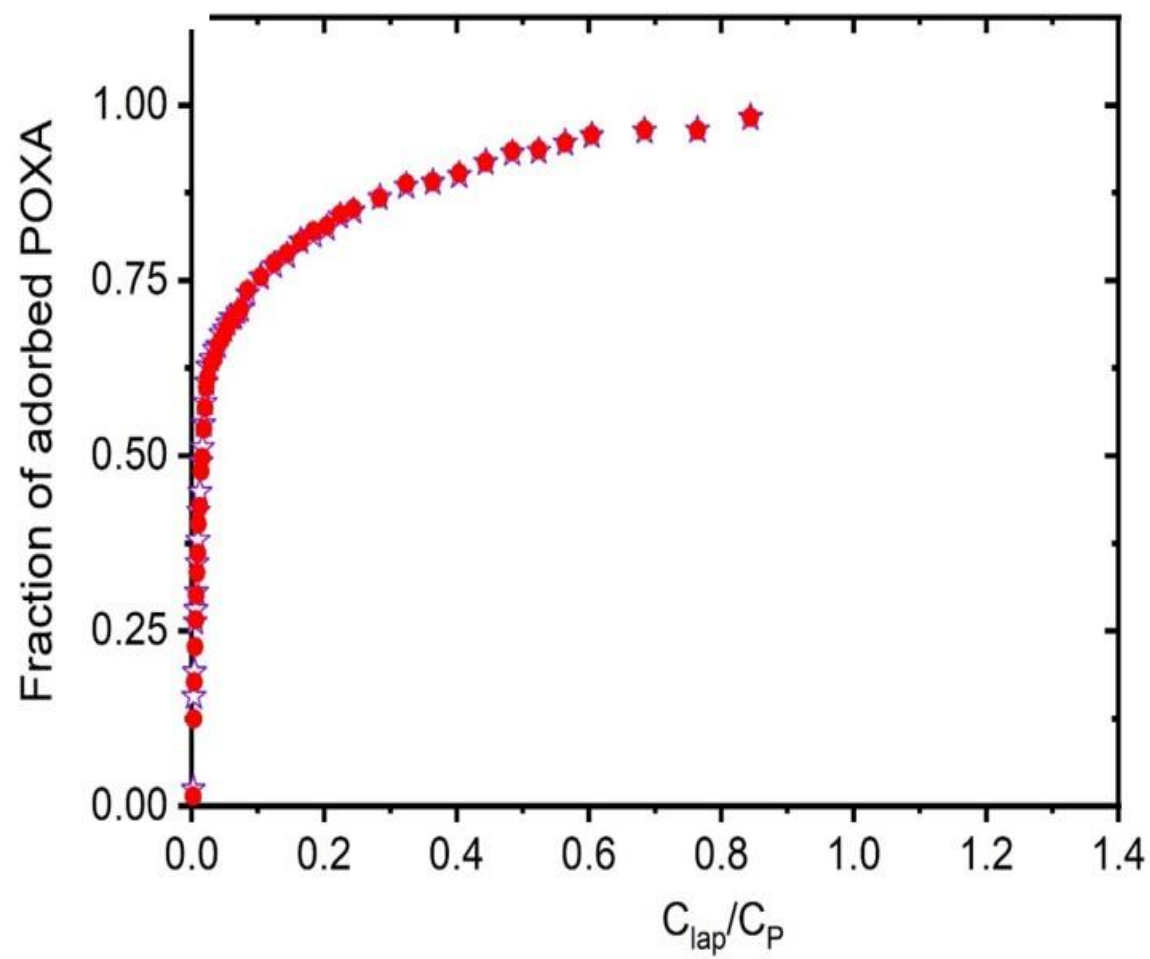


Fig. 1. (a) POXA chemical structure and ${ }^{1} \mathrm{H}$ NMR spectra of POXA (initial concentration $c_{p}$ of 20 g. $\mathrm{L}^{-1}$ ) in $\mathrm{D}_{2} \mathrm{O}$, obtained upon successive addition of a Laponite dispersion : $c_{l a p} / c_{p}=0.006$ (brown line), 0.012 (yellow line), 0.140 (green line), 0.250 (light blue line), 0.830 (dark blue line) and 1.250 (purple line), (b) Fraction of adsorbed POXA units on the clay surfaces (trains) as a function of the ratio $\mathrm{c}_{\mathrm{lap}} / \mathrm{c}_{\mathrm{p}}$. This fraction was determined by using the area under the ${ }^{1} \mathrm{H}$ NMR peaks of POXA: $-\mathrm{CH}_{3}$ peak $(\bullet)$ and $-\mathrm{CH}_{2}$ - peak $(\star)$. The error bars are smaller than the symbols.

structure (Fig. 1a). Indeed, due to the electron delocalization over the N-C-O region, the $\mathrm{N}(\mathrm{C}=\mathrm{O}) \mathrm{C}$ entities are locally planar and the only degree of freedom, beyond the conformational motions along the POXA main chain and the $-\mathrm{CH}_{3}$ rotation around its $\mathrm{C}_{3}$ axis, is the rotation of $\mathrm{N}(\mathrm{C}=\mathrm{O}) \mathrm{CH}_{3}$ around the $\mathrm{N}-\mathrm{CO}$ bond. The possible interactions between such planar entities along the POXA chains may help promoting train-like conformations.

As the POXA chain portions adsorbed at the Laponite surfaces are not detected on the ${ }^{1} \mathrm{H}$ NMR spectrum, these NMR measurements can be used to quantify the adsorption behaviour. The evolution of the ${ }^{1} \mathrm{H}$ NMR spectrum was thus monitored upon the progressive addition of small amounts of Laponite dispersion at $1 \mathrm{wt} \%$ in a solution of POXA in $\mathrm{D}_{2} \mathrm{O}$. Data were collected in order to cover the desired $c_{\mathrm{lap}} / \mathrm{c}_{\mathrm{p}}$ range and the initial POXA concentration was fixed at $2 \mathrm{wt} \%$. This concentration was chosen in order to stay in the same region of the phase diagram reported for Laponite/water systems $[4,29]$ and, in particular, to ensure that the structure formed by the clay layers remains the same despite the concentration change resulting from the mixing with the initial POXA solution. The amount of adsorbed POXA repeat units has been determined by monitoring the reduction of both $\mathrm{CH}_{2}$ and $\mathrm{CH}_{3}$ peak areas, as depicted in Fig. 1. At this stage, it is important to note that as Laponite is added, the amplitude of the ${ }^{1} \mathrm{H}$ NMR 
peaks is strongly reduced whereas no variation of the line shape and in particular, no peak broadening, is detected (see Fig. 1a). This feature indicates that no exchange occurs between adsorbed POXA units and the ones far from the Laponite platelets, over the characteristic time scale of the experiment. As a result, the signals from POXA units observed on the ${ }^{1} \mathrm{H}$ NMR spectra exclusively arise from free polymer chains, in excess, which are not adsorbed on the clay platelets. The area of these peaks allows the fraction of immobilized POXA repeat units to be calculated for each Laponite/polymer ratio, as shown in Fig. 1b. As expected, the evolution determined using the POXA methyl protons (Fig. 1b, red circles) is found to superimpose to the one obtained with the POXA $\mathrm{CH}_{2}$ protons (Fig. 1b, blue stars). At low $\mathrm{c}_{\text {lap }} / \mathrm{c}_{\mathrm{p}}$ values, a strong adsorption of the POXA chains on Laponite is clearly observed. Total adsorption of POXA at the clay surfaces is reached as $c_{l a p} / c_{p}=0.85$. Such results are typical of high affinity isotherms [56]. By using a specific surface of around $750 \mathrm{~m}^{2} / \mathrm{g}$ for Laponite [30,42,57], the adsorbed amount of POXA at saturation can be estimated to Qs $=1.56 \mathrm{mg} / \mathrm{m}^{2}$. For PEO with the same degree of polymerization, a value at least three times weaker was obtained $\left(\mathrm{Qs}=0.58 \mathrm{mg} / \mathrm{m}^{2}\right.$ [42], or Qs $=0.163 \mathrm{mg} / \mathrm{m}^{2}$ [47]). A similar extent of adsorption on clays for PEO was achieved for PEO chains with a higher molecular weight (20 000 g.mol $\left.{ }^{-1}\right)$ [42]. In the present case, at saturation, the number of POXA chains is thus estimated to 560 chains per clay platelet (280 on each face). Taking into account the radius of gyration of POXA with $M_{\mathrm{n}}=3400 \mathrm{~g} \cdot \mathrm{mol}^{-1}$ to be around $2 \mathrm{~nm} \mathrm{[58],} \mathrm{this} \mathrm{estimate} \mathrm{suggests} \mathrm{that} \mathrm{the} \mathrm{clay}$ platelets, characterized by a diameter of about $30 \mathrm{~nm}$, are densely covered by POXA chains.

\subsection{Rheological measurements}


The POXA adsorption on Laponite clearly displays a distinct behaviour from PEO. Such differences may also be correlated to variations in the rheological properties of these hydrogels with respect to Laponite/PEO systems. Shear-stress-amplitude sweep measurements were carried out at a frequency of $1 \mathrm{~Hz}$ on Laponite/POXA hydrogels prepared with a fixed Laponite concentration $(2 \mathrm{wt} \%)$ and various polymer concentrations ranging between 0.21 and $1.89 \mathrm{wt} \%$. In the POXA concentration range investigated, the polymer chains are mostly adsorbed on the clay layers and there should not be any free chains in solution as $\mathrm{c}_{\mathrm{lap}} / \mathrm{c}_{\mathrm{p}}$ is always larger than 0.85 (see Section 3.1). Fig. 2 displays the results obtained after 2 days following the gel preparation. For the sake of clarity, only the storage modulus $\mathrm{G}^{\prime}$ is reported in this figure. The same trends have been qualitatively observed after 1 day or 11 days (Supplementary Material, Fig. S2). The evolution of $\mathrm{G}^{\prime}$ with the oscillation amplitude shows the same shape, whatever the POXA concentration: a plateau of $\mathrm{G}^{\prime}$ (with $\mathrm{G}^{\prime}>\mathrm{G}^{\prime \prime}$ ) until a stress amplitude ranging from 8 to $80 \mathrm{~Pa}$ is achieved and above this value, the gels break. 


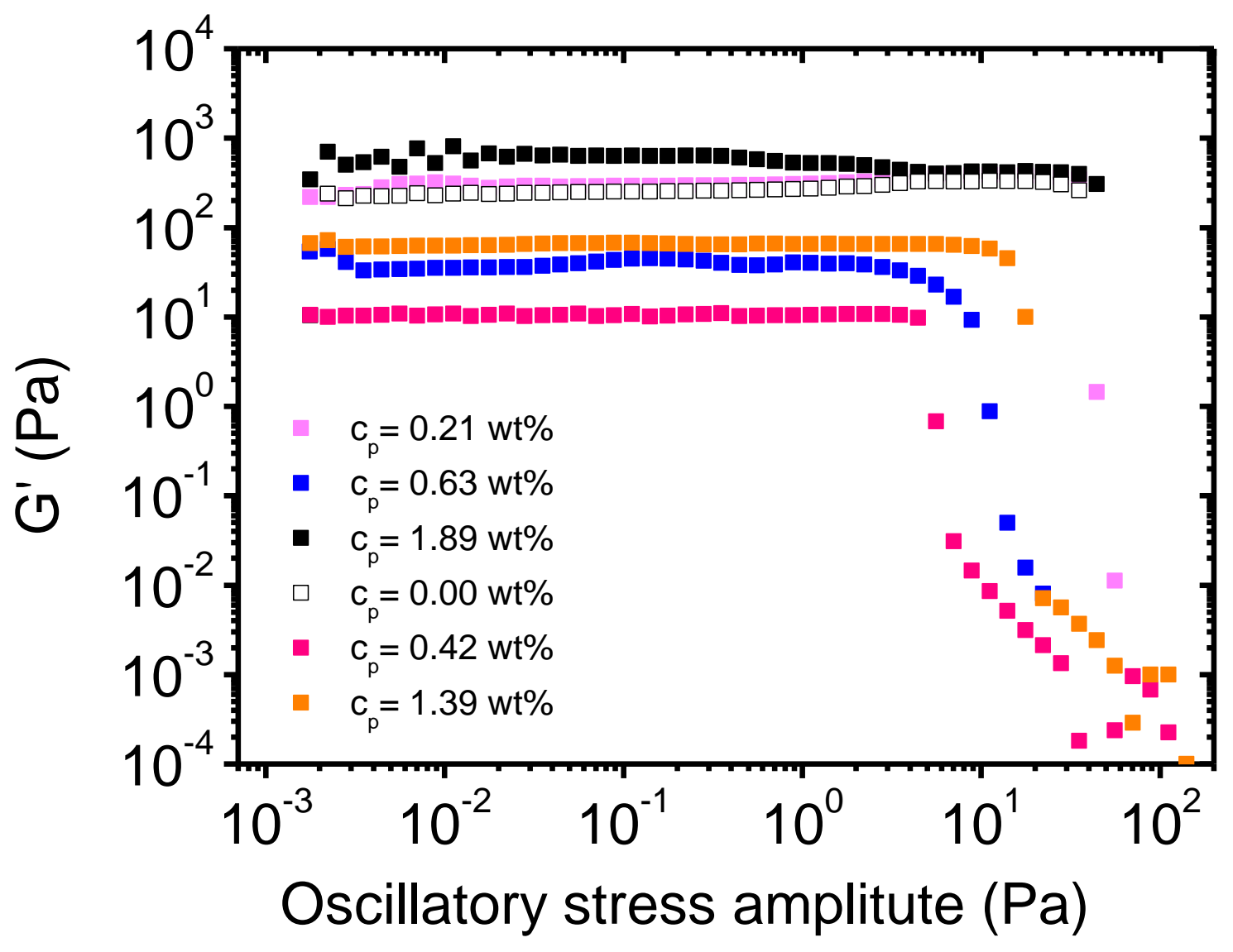

Fig. 2. Storage shear moduli se as a function of the oscillatory stress amplitude for different Laponite/POXA hydrogels. All the measurements were performed at $1 \mathrm{~Hz}$. The Laponite weight percent has been fixed to $2 \mathrm{wt} \%$ while the POXA concentration has been varied: $0 \mathrm{wt} \%(\square)$, $0.21 \mathrm{wt} \%(\square), 0.42 \mathrm{wt} \%(\square), 0.63 \mathrm{wt} \%(\bullet), 1.39 \mathrm{wt} \%(\square)$, and $1.89 \mathrm{wt} \%$ (ロ). The error bars are smaller than the symbols.

For low POXA concentration values (below $0.63 \mathrm{wt} \%$ ), the plateau value of $\mathrm{G}^{\prime}$ decreases upon raising up the polymer concentration. For $\mathrm{c}_{\mathrm{p}}=0.63 \mathrm{wt} \%, \mathrm{G}^{\prime}$ is found to be almost 100 times weaker than the one of a neat Laponite gel, while the reduction factor is equal to 20 only for PEO (Supplementary Material, Fig. S3). Such a trend has already been investigated $[42,47]$ for Laponite/PEO hydrogels. For these latter, the reduction of the modulus $\mathrm{G}^{\prime}$ is interpreted as 
resulting from the effect of the PEO adsorption on the clay platelets, which screens the electrostatic interactions between the clay layers. Laponite is indeed composed of charged disks and the gelation of Laponite aqueous dispersions at such concentrations is mainly driven by the electrostatic interactions. In the case of POXA, ${ }^{1} \mathrm{H}$ NMR experiments (Section 3.1) indicated that the POXA adsorption on Laponite is stronger than the one of PEO. The amount of adsorbed POXA chains on the Laponite disks is three times higher than the one obtained for PEO chains with a similar degree of polymerization. Therefore, one may expect a more efficient screening of the electrostatic interactions between the clay platelets for POXA than for PEO and the formation of weaker gels. This feature rationalizes the more pronounced reduction of the plateau value of G' observed with POXA, compared to PEO.

Moreover, interestingly, beyond $c_{p}=0.63 \mathrm{wt} \%$, the plateau modulus increases with the POXA concentration and even reaches, for $c_{p}=1.89 \mathrm{wt} \%$, a $\mathrm{G}^{\prime}$ value similar to the one of a neat Laponite gel. These results are reported on Fig. 3 and the data determined for Laponite/PEO hydrogels with PEO chains of the same degree of polymerization have also been included in the plot, for the sake of comparison. For the POXA/Laponite hydrogels, the dependence with $c_{p}$ of the plateau modulus $G^{\prime}$ exhibits an asymmetric U-shape curve. Such a behaviour completely differs from the one obtained for the PEO/Laponite hydrogels for which G' continuously decreases upon addition of polymer chains during the gel formation. As it will be discussed in the next section (structural studies of the Laponite/POXA hydrogels), the variation of $G^{\prime}$ with $c_{p}$ above $0.63 \mathrm{wt} \%$ may be due to increased steric repulsions between the clay platelets. 


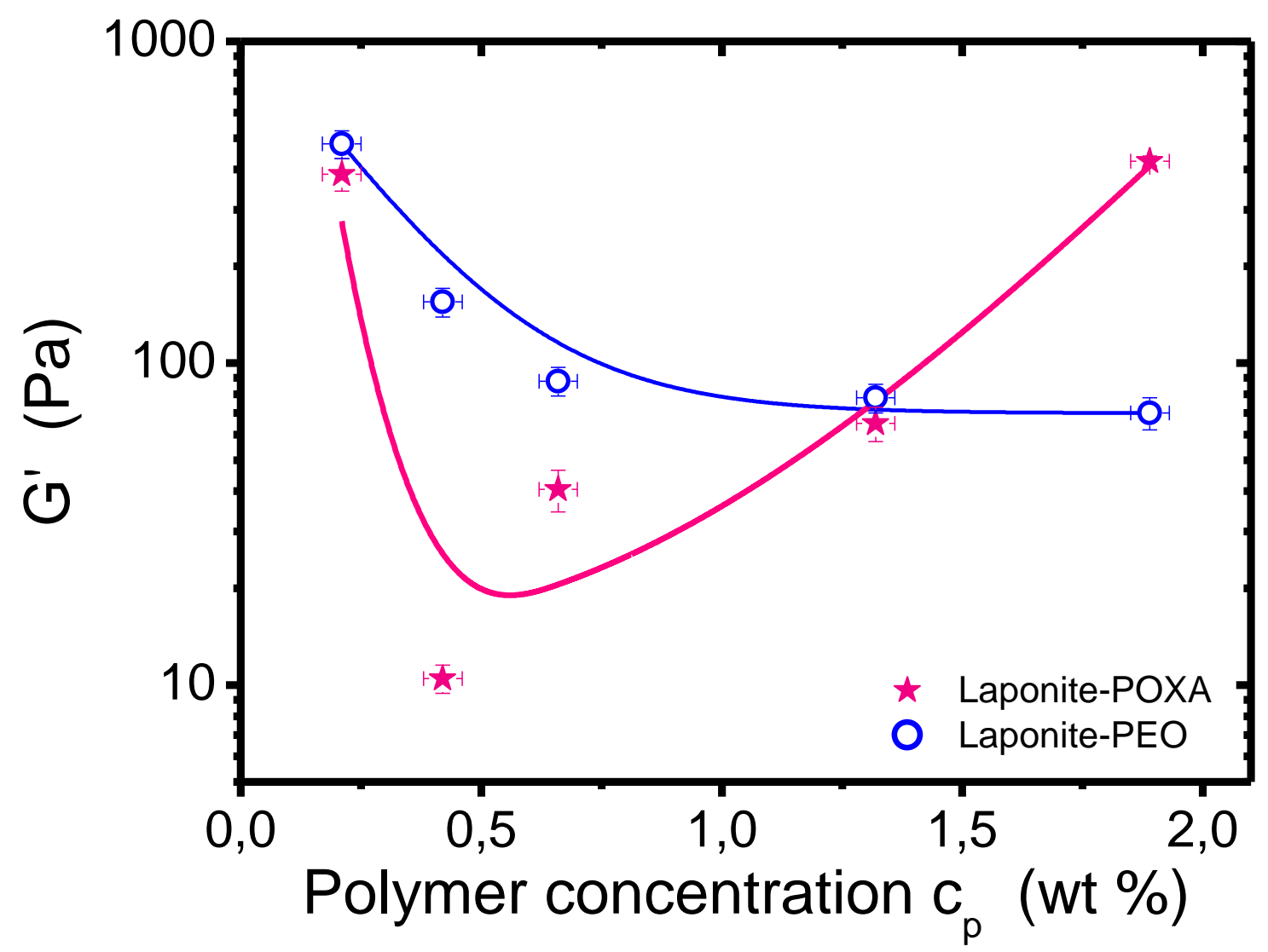

Fig. 3. Plateau modulus as a function of the polymer weight fraction, for different Laponite/polymer hydrogels: Laponite/POXA (O), Laponite/PEO ( $\star$ ). The Laponite concentration was set to $2 \mathrm{wt} \%$ and both series of hydrogels were prepared using the very same experimental conditions. Solid lines serve as guides for the eyes.

Similar measurements have been performed for different values of the Laponite concentration: $1.5 \mathrm{wt} \%$ and $2.5 \mathrm{wt} \%$. These latter have been chosen in order to remain in the same part of the Laponite/water phase diagram as for $\mathrm{c}_{\mathrm{lap}}=2.0 \mathrm{wt} \%$ (isotropic gel). The POXA concentration was varied from $0.21 \mathrm{wt} \%$ to $1.80 \mathrm{wt} \%$, as previously, ensuring that POXA chains are mostly adsorbed on the clay disks, except for the highest concentration of $1.80 \mathrm{wt} \%$. At these concentrations, Laponite/POXA also form 
hydrogels and the oscillation amplitude sweep tests performed 2 days after the sample preparation follow the same trends as the ones obtained for $c_{l a p}=2 \mathrm{wt} \%$ : $\mathrm{G}^{\prime}$ is higher than $G^{\prime}$, exhibits a plateau for low stress amplitudes and significantly decreases above a certain amplitude. The 'evolution of the plateau modulus with $c_{p}$ for both Laponite concentrations (1.50 wt \% and $2.50 \mathrm{wt} \%)$ are reported in Fig. S4. In the absence of POXA, the modulus increases with $\mathrm{c}_{\text {lap }}$, as already reported in the literature $[20,27]$. Both $\mathrm{G}^{\prime}\left(\mathrm{c}_{\mathrm{p}}\right)$-dependences observed for $\mathrm{c}_{\mathrm{ap}}=1.50 \mathrm{wt} \%$ and $2.50 \mathrm{wt} \%$ are qualitatively similar to the one derived for $\mathrm{c}_{\mathrm{lap}}=2.0 \mathrm{wt} \%$ with, in particular, the U-shape. Interestingly, the critical concentration $c_{p}$ corresponding to the minimum of the U-shape curves seems to be nearly unchanged (about $0.6 \mathrm{wt} \%$ ), within the experimental accuracy, in the clap range investigated. Taking into account the previous result that full platelet coverage is obtained for $\mathrm{c}_{\mathrm{lap}} / \mathrm{c}_{\mathrm{p}} \sim 0.85$, the polymer coverage of the platelets at the $\mathrm{G}^{\prime}$ minima evolves from 20 to $34 \%$ when the clay concentration is decreased from 2.5 to $1.5 \mathrm{wt} \%$. These results suggest that the antagonistic effects at the origin of the U-shape of $G^{\prime}\left(c_{p}\right)$ should be sensitive to the Laponite concentration. Whereas the screening of the electrostatic interactions between the platelets should decrease similarly with $c_{p}$ whatever $c_{\text {lap }}$ in the probed range, one expects that the contribution of steric repulsions between platelets gets stronger as $c_{l a p}$ increases. This could explain the occurrence of $G^{\prime}$ minimum values at lower platelet coverage when $c_{\text {lap }}$ gets higher.

\subsection{Small-Angle Neutron Scattering}

\subsubsection{Scattering from Laponite dispersions with absorbed POXA}

SANS measurements have been performed on hydrogels prepared and aged under the same conditions as for the rheological experiments. More precisely, all the samples 
were measured two days after their preparation. The scattering intensity from a $2 \mathrm{wt} \%$ Laponite dispersion in $25 \mathrm{wt} \%$ of $\mathrm{D}_{2} \mathrm{O}$ is shown in Fig. 4. The incoherent background has been subtracted. I(q) displays a q $\mathrm{q}^{-2}$ dependency at high q-values (beyond $0.02 \AA^{-1}$ ), characteristic of the discoidal shape for the platelets of such clays. The scattered intensity can be fitted by the form factor of a disk (Eq. (4)), the corresponding radius of the particles was estimated to $148 \pm 3 \AA$ and the thickness, to $10 \pm 1 \AA$. These results are consistent with previous data reported for Laponite [26-28].

For the Laponite/POXA hydrogels, the contribution from POXA chains to the scattered intensity can be matched by the solvent, using a mixture $\mathrm{H}_{2} \mathrm{O} / \mathrm{D}_{2} \mathrm{O}$ with $25 \mathrm{wt} \%$ of $\mathrm{D}_{2} \mathrm{O}$ (see Section 2.5, "Materials and Methods"). Under this condition, $\rho_{\mathrm{p}}=\rho_{\mathrm{s}}$ in Eq. (1). Thus, with $25 \%$ of $\mathrm{D}_{2} \mathrm{O}$, the scattered intensity only results from the contribution of Laponite layers. The curves I(q) of Laponite-based gels at a clay concentration of $2 \mathrm{wt} \%$ in presence of POXA at various concentrations are reported in Fig. 4. For q $>4 \cdot 10^{-3} \AA^{-}$ 1, all the data superimpose on the same profile. The scattered intensities for Laponite within the composite hydrogels are the same as the one without POXA chains and the same discoidal shape could fit all the data. At very small q-values (below $4.10^{-3} \AA^{-1}$ ), weak but detectable differences between the different I(q) curves may be observed. At low POXA concentrations $\left(c_{p}=0.42 \mathrm{wt} \%\right.$ and $\left.c_{p}=0.66 \mathrm{wt} \%\right)$, the slope of $\mathrm{I}(\mathrm{q})$ below $\mathrm{q}$ $=4.10^{-3} \AA^{-1}$ is slightly higher than the one observed for higher POXA concentrations $\left(c_{\mathrm{p}}\right.$ $=1.20 \mathrm{wt} \%$ and $\left.\mathrm{c}_{\mathrm{p}}=1.60 \mathrm{wt} \%\right)$. This feature suggests the presence of more aggregates or inhomogeneities for clays in the hydrogels as the POXA concentration is low enough. These aggregates or inhomogeneities could correspond to a small amount of remaining tactoids and/or to structural inhomogeneities at larger scale. Their contribution is found to decrease as the amount of POXA increases. 


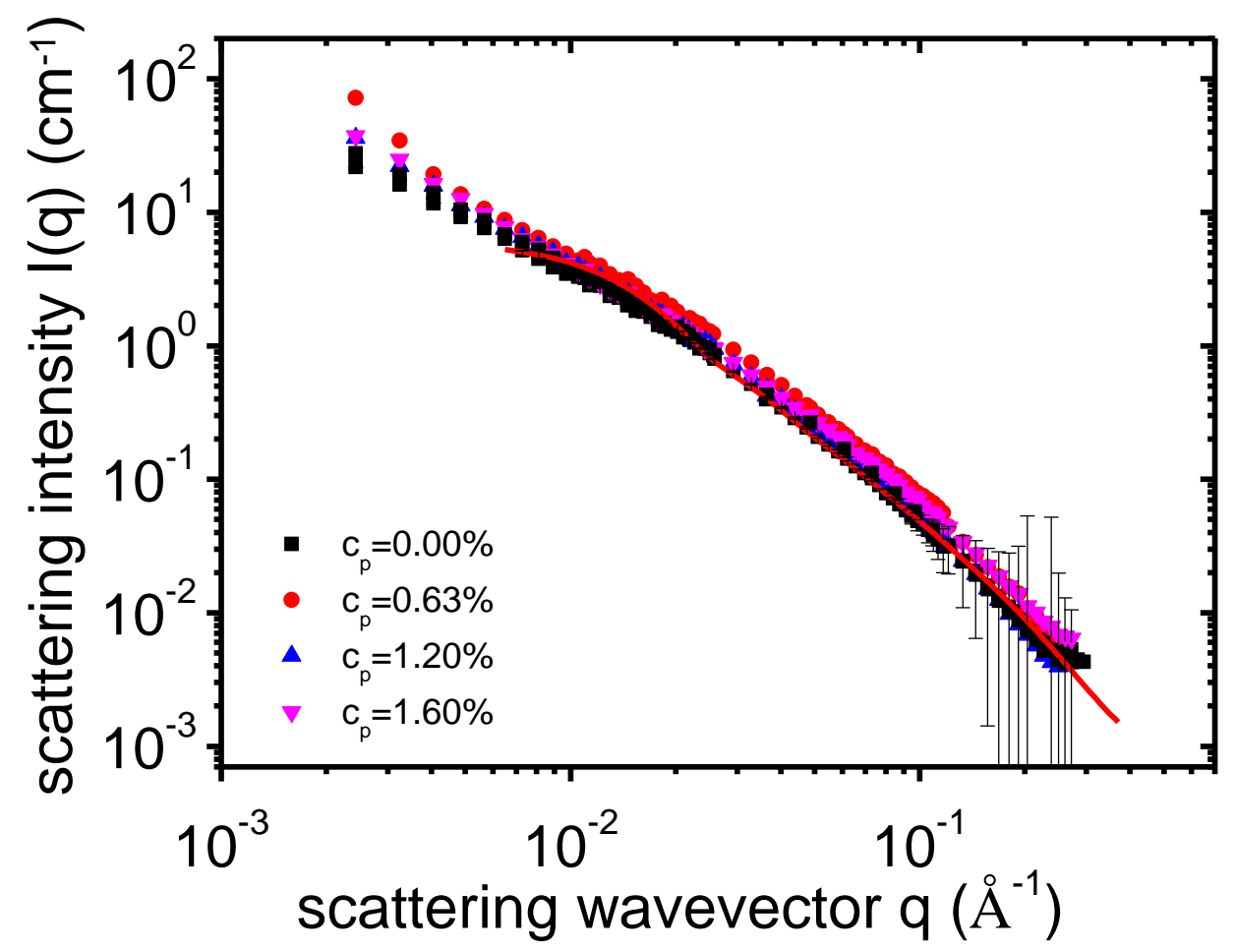

Fig. 4. Scattered intensity of Laponite as a function of the wavevector for Laponite/POXA systems with different values of the polymer concentration, $\mathrm{c}_{\mathrm{p}}$, ranging from 0 to $1.60 \mathrm{wt} \%: \mathrm{c}_{\mathrm{p}}=0 \mathrm{wt} \%$ in black, $0.63 \mathrm{wt} \%$ in red, $1.20 \mathrm{wt} \%$ in blue and 1.60 wt $\%$ in pink. The Laponite concentration is fixed at $2 \mathrm{wt} \%$. The weight percentage of $\mathrm{D}_{2} \mathrm{O}$ in the solvent $\left(\mathrm{D}_{2} \mathrm{O} / \mathrm{H}_{2} \mathrm{O}\right.$ mixture $)$ is set at $25 \%$, so that the contribution of POXA to $\mathrm{I}(\mathrm{q})$ is matched and the scattering intensity only results from the Laponite disks. The error bars are represented only for one hydrogel $\left(c_{p}=0 \mathrm{wt} \%\right)$ for the sake of clarity. The experimental accuracy stands in the same order of magnitude for the other hydrogels. The best fit of the experimental data above $\mathrm{q}=0.006 \AA$, obtained with a discoidal shape (Eq. (4)), is represented as a red solid line.

\subsubsection{Scattering from POXA in Laponite/POXA hydrogels}


Laponite can be matched using a $\mathrm{H}_{2} \mathrm{O} / \mathrm{D}_{2} \mathrm{O}$ mixture with 68 wt $\%$ of $\mathrm{D}_{2} \mathrm{O}$. In Fig. S5, the scattered intensity of a Laponite dispersion at $2 \mathrm{wt} \%$ in such a solvent mixture, without POXA, is shown (black symbols). The intensity profile is completely flat and corresponds to the incoherent scattering due to the solvent. On the same plot, the scattered intensity at the same Laponite concentration is reported for different POXA concentrations. Reassuringly, the corresponding curves, including the one obtained for $c_{p}=0 \%$, superimpose for high q-values, since all of them tend to the incoherent background level.

Let us now focus on the POXA conformation within the Laponite/POXA hydrogels. The polymer concentration was restricted to be lower than $c_{l a p}$, ensuring that all the POXA chains are adsorbed at the clay surface (see Section 3.1). Scattering intensity increases with the concentration of scattering species (here, POXA chains under the matching conditions used), as expected from Eq. (2). Therefore, in order to remove this direct influence of the polymer concentration on $\mathrm{I}(\mathrm{q})$, all the data were divided by $\mathrm{c}_{\mathrm{p}}$ and the solvent background scattering was removed, as depicted in Fig. 5. It is worth remarking that all the thus-normalized data lie on the same "master curve" (Fig. 5a). This result clearly indicates that the conformational behaviour of the POXA chains in the hydrogels remains the same, whatever the polymer concentration in the regime $c_{p}<$

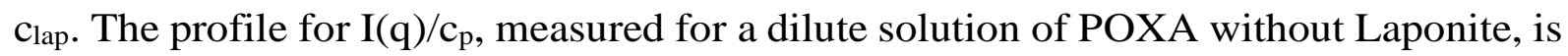
also reported in Fig. 5 (green curve, $\mathrm{c}_{\mathrm{p}}=1.4 \mathrm{wt} \%$ ). The form factor of POXA in solution can be fitted by the Debye function for Gaussian polymer chains (Eq. (3)). The radius of gyration of POXA, deduced from this fit, is found to be $\mathrm{R}_{\mathrm{g}, \mathrm{p}}=21 \AA$, which is in good agreement with the expected value for a chain with this molecular weight in good solvent [58]. In contrast, the form factor of POXA chains in the Laponite-based hydrogels strongly differs from the one of free POXA chains. Indeed, the single-chain form factor 
for Gaussian chains cannot describe satisfactorily the polymer form factors experimentally measured in these systems, as shown in Fig. 5. 
(a)

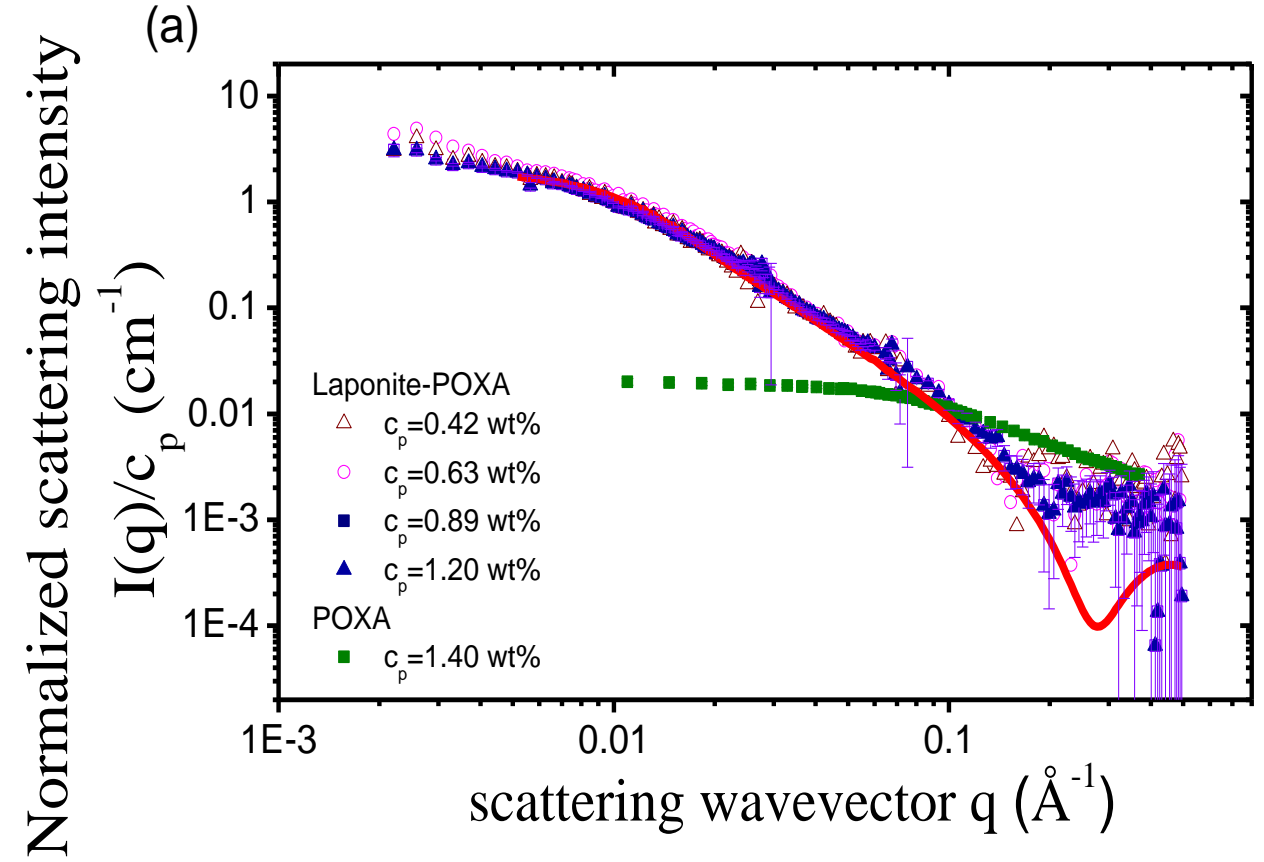

(b)

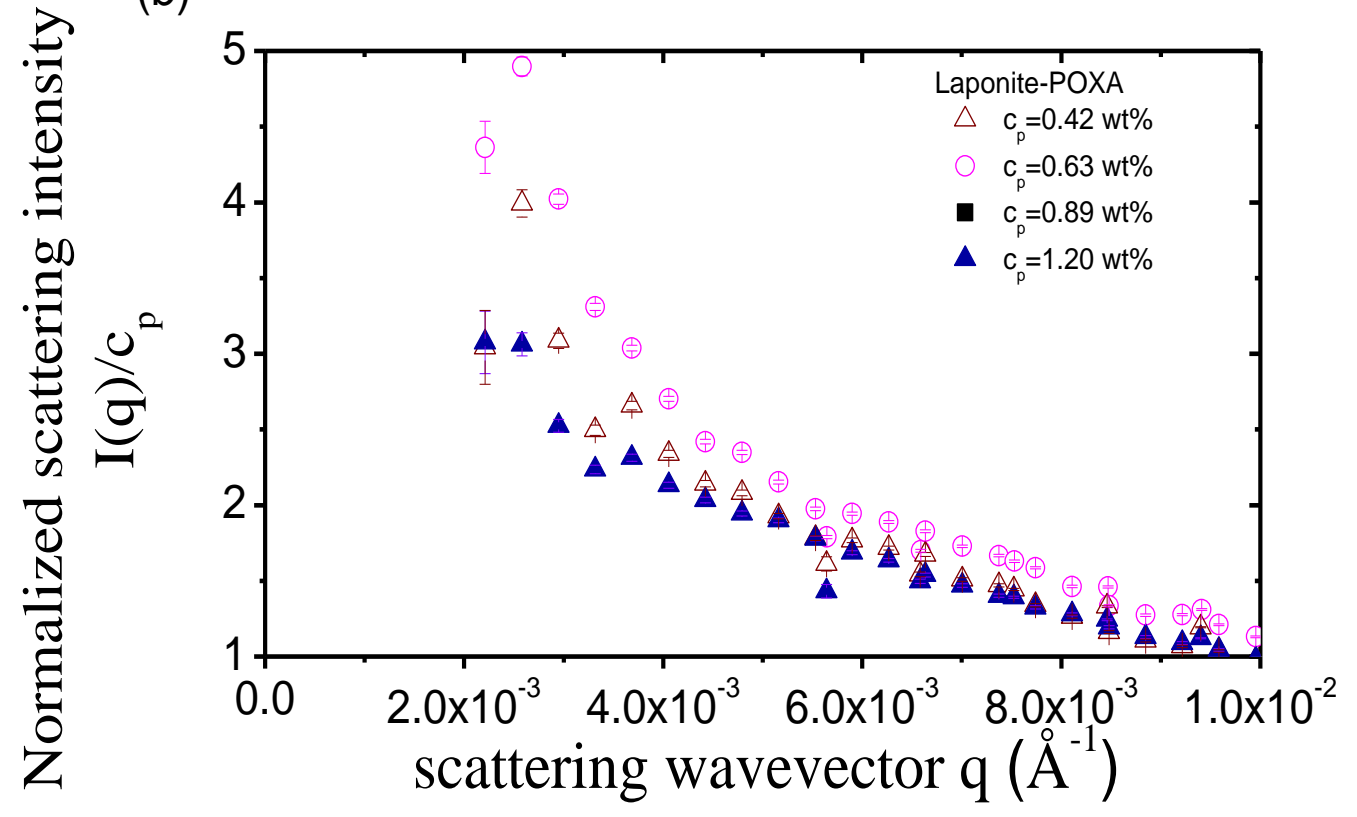

Fig. 5. (a) Scattered intensity $I(q)$, normalized by the POXA concentration $c_{p}$, as a function of the wavevector q for Laponite/POXA samples with different polymer concentrations: $c_{p}=0.42$ $(\triangle), 0.63(0), 0.89(\square)$ and $1.20(\triangle) w t \%$. The Laponite concentration is fixed at $1.5 \mathrm{wt} \%$. The proportion of $\mathrm{D}_{2} \mathrm{O}$ in the mixture $\mathrm{H}_{2} \mathrm{O} / \mathrm{D}_{2} \mathrm{O}$ is set to $68 \mathrm{wt} \%$ so that Laponite is matched and the scattered intensity is only due to POXA. The scattering intensity of a solution of POXA 
at $1.4 \mathrm{wt} \%$ in $\mathrm{D}_{2} \mathrm{O}$ is also added ( $\left.\square\right)$, for the sake of comparison. The error bars are represented only for one hydrogel $\left(c_{p}=1.20 \mathrm{wt} \%\right)$ for the sake of clarity. The experimental accuracy stands in the same order of magnitude for the other hydrogels. (b) Zoom of the scattered intensities for the same samples at small q-values is represented in a linear-linear plot.

Besides, the I(q) profile observed at small q-values for the hydrogels indicates the presence of large objects, as shown in Fig. 5b. Lastly, the slope of I(q) for high q-values displays a power-law decay, $\mathrm{I}(\mathrm{q}) \propto \mathrm{q}^{-2}$, which is characteristic of discoidal shape, though only the POXA chains (and not the Laponite layers) are observed in these contrastmatching experiments. In fact, the curves $\mathrm{I}(\mathrm{q})$ do not describe the conformation of an individual POXA chain in the hydrogels, but the global shape of the aggregates.
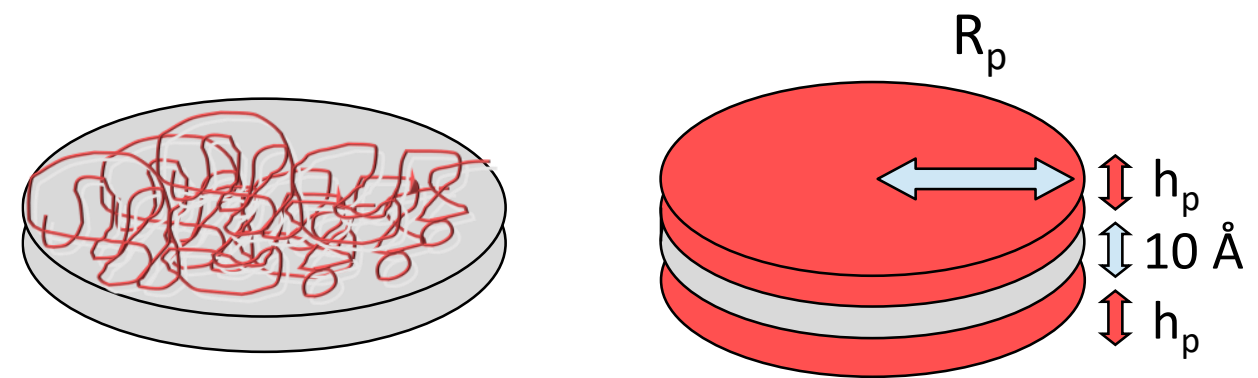

Fig. 6. Schematic representation of polymer chains flatly adsorbed on clay disks.

NMR measurements indicated that for $c_{p}<c_{l a p}$, POXA is completely absorbed on the Laponite clay platelets, most of the units being involved in train conformations. As a result, the SANS data depicted in Fig. 5a were fitted by a form factor describing two parallel layers of POXA chains flatly adsorbed on each face of the Laponite disks, as schematically represented in Fig. 6. The scattering intensity of such an object is given by the following expression [59]:

$$
I(q)=\int_{0}^{\pi / 2} \sin \theta d \theta V_{p}^{2} \Delta \rho^{2}\left[\frac{\sin \left(q \cdot h_{p} \cdot \frac{\cos (\theta)}{2}\right)}{q \cdot \frac{\cos (\theta)}{2}} \cdot \frac{2 J_{1}\left[q \cdot R_{p} \cdot \sin (\theta)\right]}{q \cdot R_{p} \cdot \sin (\theta)}\right] \cdot S(q)
$$


where $a \times b \cdot$

$$
S(q)=1+\frac{2}{n} \sum_{k=1}^{n}(n-k) \cdot \cos \left(k \cdot D \cdot q \cdot \cos (\theta) \cdot \exp \left(-\frac{k\left(q \cdot \cos (\theta) \cdot \sigma_{D}\right)^{2}}{2}\right)\right.
$$

$\Delta \rho$ corresponds to the difference of the scattering length density between polymer and solvent; $V_{p}$, to the volume of a disk of polymer; $D$ is the distance between the two polymer layers and $\sigma_{D}$, the Gaussian standard deviation for $D$. As can be seen on Fig. 5a, a very good description of the SANS data is obtained using this model and the resulting fit leads to the thickness and the radius of the adsorbed polymer layers.

For all polymer concentrations from 0.42 to $1.20 \mathrm{wt} \%$, the distance between the two layers, which is equal to $D-h_{p}$ (and which corresponds to the thickness of the clay platelet here), was found to be $9 \AA$, with $\sigma_{D}=0.1 \AA$. The radius of the adsorbed POXA layers obtained through the fitting procedure is $\mathrm{R}_{\mathrm{p}}=151 \AA$ and their thickness, $h_{p}=5 \AA$. The radius $R_{p}$ of the adsorbed disk of polymer is really close to the radius of the bare Laponite nanoparticles, which is equal to $149 \AA$. It is also worth remarking that the dimensions of scattering objects do not change with the polymer concentration (Table S1). The thickness $h_{\mathrm{p}}$ of $5 \AA$, i.e. the characteristic size of one repeat unit, is much smaller than the POXA radius of gyration in solution, without clay, and consistent with the fact that POXA does not form a significant amount of loops or tails, but mostly trains on the Laponite surface.

Extrapolating Eq. (2) as the scattering wavevector tends to zero allows the apparent mass of POXA layers at the Laponite surfaces to be estimated, using the following equation: 


$$
M_{a}=\frac{I_{(q \rightarrow 0)} N_{A}}{v_{S}^{2} \cdot c \cdot(\Delta \rho)^{2}}
$$

An apparent mass of polymer adsorbed on a clay platelet of $M_{\mathrm{a}}=952000 \mathrm{~g} \cdot \mathrm{mol}^{-1}$ was thus determined. This corresponds to a number of POXA chains per face of $N_{\mathrm{c}}=280$, which results in a dense layer of POXA chains adsorbed on the clay surface. The number of POXA chains per Laponite face derived by SANS is in good agreement with the one obtained through our NMR measurements at the plateau of the adsorption isotherm $\left(N_{\mathrm{c}}\right.$ $=280$, see Section 3.1). However, it is surprising to derive the same number of adsorbed chains per face of clay platelet, whatever the POXA concentration. If one assumes that the platelets are fully exfoliated in all the hydrogels considered and that the complete adsorption of the POXA chains onto the clay layers occurs homogeneously, then the number of chains per face should increase from 44 to 126 as the POXA concentration is raised up from 0.42 to $1.20 \mathrm{wt} \%$. In contrast, the SANS results show a saturation of adsorption in all the cases. One possible explanation of this result is that a small amount of clay platelets would not be fully exfoliated before the POXA addition. Previous lightscattering experiments performed with the same conditions of Laponite dispersion support this argument [42]. Strong POXA adsorption on the Laponite surface should promote the exfoliation of the remaining tactoids, thus resulting in an increase of the possible contact area between Laponite and POXA. Such an evolution may compensate the concomitant increase of the quantity of adsorbing polymer chains and thus leads to a nearly constant value of $M_{\mathrm{a}}$ as $\mathrm{c}_{\mathrm{p}}$ is raised up.

Similarly to the scattering curves I(q) of the clay layers within Laponite/POXA hydrogels (see Fig. 4), a careful analysis of the scattered intensities of Fig. 5a shows that at very low q-values $\left(\mathrm{q}<4.10^{-3} \AA^{-1}\right)$, the superimposition of the different curves is not valid anymore. This result may be more explicitly realized by using a linear-linear 
representation of the data, as shown in Fig. 5b. The increase of $\mathrm{I}(\mathrm{q})$ in the low-q regime is more significant at low POXA concentrations (for $0.42 \mathrm{wt} \%$ and $0.63 \mathrm{wt} \%$ ) than for the higher concentrations investigated. This feature implies that more aggregates or inhomogeneities occur for the low-concentrated samples than for the more concentrated ones.

\subsubsection{Influence of the Laponite content in the hydrogels}

In the previous section, hydrogels with an excess of Laponite were considered $\left(c_{l a p} / c_{p}\right.$ was varied from 1.2 to 3.5 ) in order to study the conformation of the adsorbed POXA chains. In order to investigate the POXA adsorption behaviour well below the saturation regime, it may be worth increasing further this ratio. However, the decrease of the polymer concentration cannot be envisioned for sensitivity reasons intrinsic to scattering experiments: the scattered intensity from POXA chains would be too low to be detected. Another way to reach this goal is to increase the Laponite weight fraction. Therefore, SANS measurements were performed at a weight percentage of Laponite fixed at $2.5 \%$. At this concentration, the Laponite/water system always stands in the same part of its complex phase diagram [4, 21, 22, 25]. The POXA concentration has been varied from 0.42 to $1.89 \mathrm{wt} \%$. As previously, the scattering intensity $\mathrm{I}(\mathrm{q})$ has been divided by the POXA concentration, $\mathrm{c}_{\mathrm{p}}$, and the incoherent scattering from the solvent has been subtracted. The resulting curves are reported in Fig. 7.

\begin{tabular}{|c|c|c|c|c|c|}
\hline $\mathrm{c}_{\mathrm{p}}(\mathrm{wt} \%)$ & 0.42 & 0.63 & 0.89 & 1.20 & 1.80 \\
\hline$M_{\mathrm{a}}\left(\mathrm{g} \cdot \mathrm{mol}^{-1}\right)$ & 185000 & 420000 & 880000 & 900000 & 952000 \\
\hline$N_{\text {ch/face }}$ & 55 & 120 & 260 & 265 & 280 \\
\hline
\end{tabular}


Table 1. Apparent molecular weight of the adsorbed POXA layer $\left(\mathrm{M}_{\mathrm{a}}\right)$ per platelet and number of chains per Laponite face $\left(N_{\mathrm{ch} / \mathrm{face}}\right) . \mathrm{c}_{\text {lap }}=2.5 \mathrm{wt} \%$.

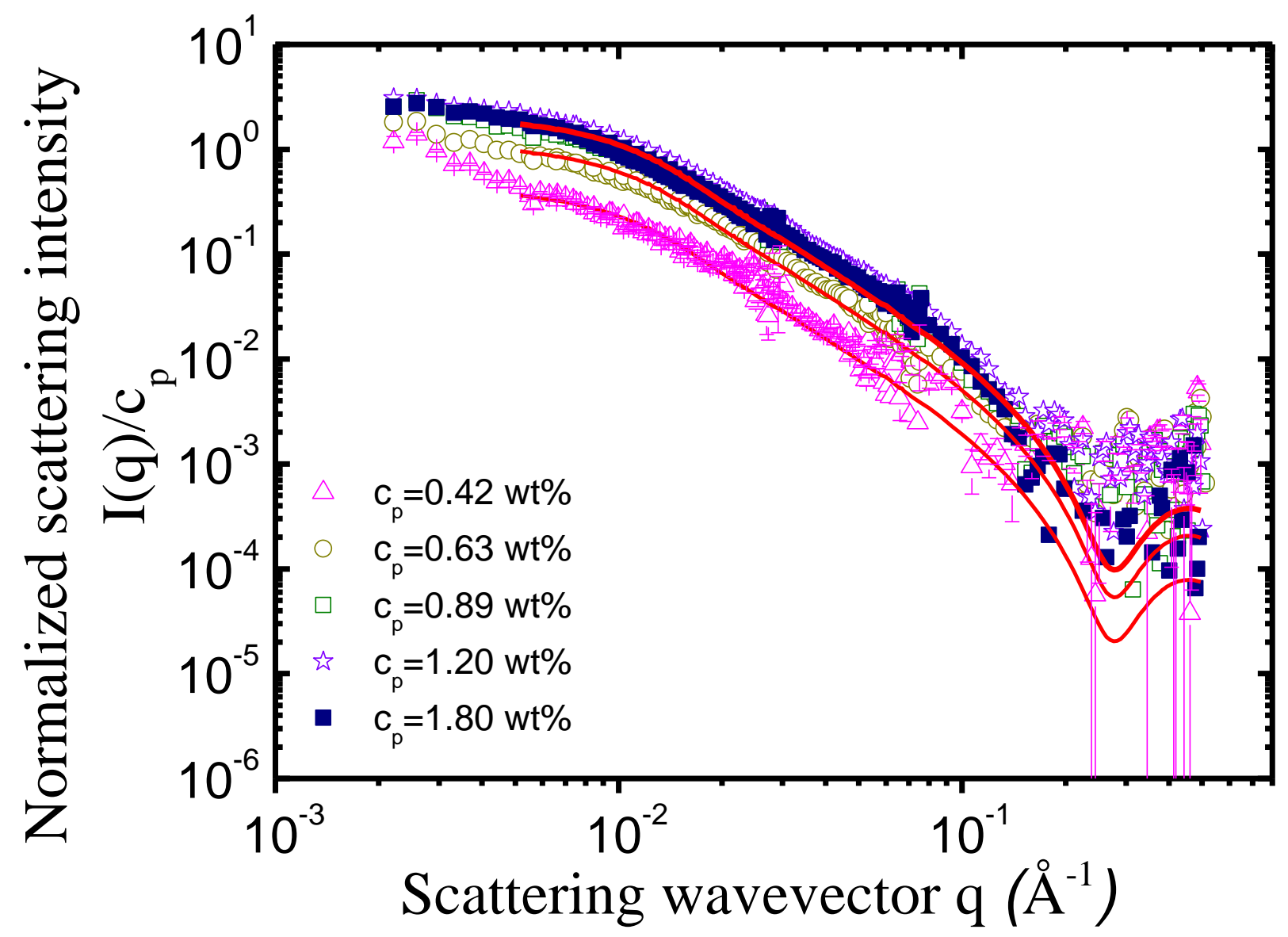

Fig. 7. Normalized scattered intensity, $\mathrm{I}(\mathrm{q}) / \mathrm{c}_{\mathrm{p}}$, of Laponite/POXA hydrogels under the Laponite matching condition. The concentration of Laponite is fixed at $2.5 \mathrm{wt} \%$. The concentration of POXA is varied from 0.42 to $1.80 \mathrm{wt} \%: c_{p}=0.42 \mathrm{wt} \%(\triangle), 0.63 \mathrm{wt} \%$ (O), $0.89 \mathrm{wt} \%(\square), 1.20 \mathrm{wt} \%(\star), 1.80 \mathrm{wt} \%(\square)$. The red solid lines stand for the fits of the experimental data using the form factor expressed in Eq. (5). The error bars are 
represented only for one hydrogel $\left(c_{p}=0.42 \mathrm{wt} \%\right)$ for the sake of clarity. The experimental accuracy stands in the same order of magnitude for the other hydrogels.

As previously observed for $\mathrm{c}_{\text {lap }}=1.5 \mathrm{wt} \%$, all the curves $\mathrm{I}(\mathrm{q})$ may be satisfactorily described using the form factor given by Eq. (5) and the same values were obtained for the fitting parameters, i.e. the radius and the thickness of the adsorbed POXA layers: $R_{p}$ $=151 \AA, h_{p}=5 \AA$. The only difference stands in the normalized scattering intensity $\mathrm{I}_{(\mathrm{q} \rightarrow 0)} / \mathrm{c}_{\mathrm{p}}$ obtained as $\mathrm{q}$ tends to zero, which is proportional to the apparent mass $\left(M_{\mathrm{a}}\right)$ of the scattering objects (Eq. (6)). For the lowest polymer concentrations investigated $\left(\mathrm{c}_{\mathrm{p}}\right.$ $=0.42 \mathrm{wt} \%$ and $0.63 \mathrm{wt} \%), \mathrm{I}_{(\mathrm{q} \rightarrow 0)} / \mathrm{c}_{\mathrm{p}}$ is significantly reduced compared to the other concentrations, as can be seen in Fig. 7 and on Table S2. This result implies that the amount of adsorbed POXA chains per Laponite particle gets weaker as $c_{p}$ decreases below $0.63 \mathrm{wt} \%$. The values of $M_{\mathrm{a}}$ for the different POXA concentrations considered are collected in Table 1. At low concentrations, $c_{p}=0.42 \mathrm{wt} \%$ and $c_{p}=0.63 \mathrm{wt} \%$, lower $\mathrm{M}_{\mathrm{a}}$ values were indeed obtained: $M_{\mathrm{a}}=185000 \mathrm{~g} \cdot \mathrm{mol}^{-1}$ and $M_{\mathrm{a}}=420000 \mathrm{~g} \cdot \mathrm{mol}^{-1}$, corresponding to a number of POXA chains per platelet face of 55 and 120, respectively. Fig. 7 also shows that above a certain concentration (between 0.63 and 0.89 wt $\%$ ), the normalized scattering profile, $\mathrm{I}(\mathrm{q}) / \mathrm{c}_{\mathrm{p}}$, remains identical and, in particular, $M_{\mathrm{a}}$ does not increase anymore. This limit value is approximately the same as the one determined for POXA/Laponite hydrogels with a clay content of 1.5 wt\% (see Fig. 5). This analysis proves that the thickness and the radius of the layers of adsorbed POXA chains do not change as $\mathrm{c}_{\mathrm{lap}} / \mathrm{c}_{\mathrm{p}}$ varies from 5.9 to 1.4 whereas the number of chains per Laponite face increases with the POXA concentration until a maximum is reached around $\mathrm{c}_{\mathrm{p}}=0.63$ wt\%. Such a situation is schematically depicted in Fig. 8: in an initial stage, the density of POXA within the layers of chains adsorbed at the Laponite surface increases as the 
aqueous solution of POXA is added to the clay dispersion in water. In a second stage, the POXA density levels off.

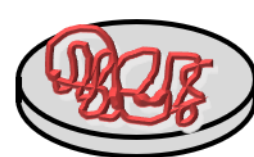

$\mathrm{c}_{\mathrm{p}} \sim 0.42 \mathrm{wt} \%$

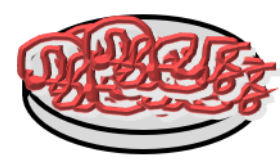

$\mathrm{c}_{\mathrm{p}} \sim 0.63 \mathrm{wt} \%$

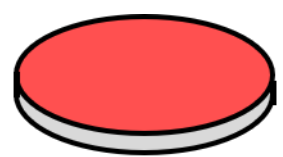

$\mathrm{c}_{\mathrm{p}} \sim 0.89-1.8 \mathrm{wt} \%$

\section{Polymer concentration}

Fig. 8. Schematic representation of the progressive densification of the adsorbed POXA chains on the Laponite disk surfaces. The thickness and the radius of the adsorbed layers do not change with the POXA concentration whereas the density of polymer increases and then levels off as $c_{p}$ is raised up. The $c_{p}$ concentration ranges are given for $c_{\text {lap }}=2.5$ $\mathrm{wt} \%$.

In this work, the adsorption of POXA onto Laponite has been investigated by the combination of NMR and SANS measurements. Both experimental approaches evidence a markedly different behaviour compared to PEO. The affinity between the POXA chains and the clay layers is found to be much stronger than the one between PEO chains and Laponite. Besides, the adsorbed chains display flat conformations, with a thickness for the absorbed layers that approximately corresponds to the characteristic size of a 
monomer. This behaviour differs significantly from Laponite/PEO hydrogels, for which PEO chains form a non-negligible fraction of loops and tails. This strong adsorption observed for POXA chains leads to a more efficient screening of the attractive interactions between clays, compared to PEO. From a macroscopic point of view, this results in a strong decrease of the plateau modulus G', about a factor of 100 in comparison to the one obtained for a Laponite hydrogel without any POXA chain. Comparing these results with the ones obtained for PEO $[18,26]$, this strong reduction is due to a stronger polymer adsorption onto the clay surfaces.

For POXA concentrations higher than a threshold of about $0.6 \mathrm{wt} \%$, a change in the evolution of the plateau modulus with the polymer concentration is detected: $G^{\prime}$ increases with $c_{p}$. This change in the macroscopic behaviour does not correspond to any structural modification of POXA at the clay surfaces, but to fluctuations of the Laponite (POXA) local concentration within the hydrogel or to platelet aggregates. The contribution of these inhomogeneities or aggregates to the SANS signals is larger at low POXA concentrations but decreases as the polymer concentration is raised up. Besides, the absence of a correlation peak in the SANS data at high q-values proves that the fraction of stacked disks and/or the number of disks within these tactoids is low. Nevertheless, Laponite layers in water are never completely exfoliated. It has been previously shown that Laponites dispersions $[42,60]$, not only contain a distribution of individual particles but also small platelets aggregates and a small fraction of very large aggregates. Even in quite well dispersed systems, the presence of small stacked clays could be detected. The dispersion of Laponite depends also on the ionic strength of the solution. The increase of salinity promotes the aggregation of clays. In the present study, at low ionic strength, such an aggregation effect induced by salt should not occur and Laponite suspensions are stabilized by repulsive electrostatic interactions whereas small 
stacks of platelets should coexist with well dispersed platelets. The adsorption of POXA on clay layers could promote the exfoliation of part of the stacked disks and thus, could increase the total amount of surface area available. The combination of both effects (complete exfoliation of some remaining clay tactoids and homogenization of the clay dispersion in solution) may account for the increase of the plateau modulus $G^{\prime}$ with $c_{p}$. Both phenomena contribute to form a gel caused by steric repulsions. Thus, POXA helps breaking electrostatic gels to form steric gels. These two antagonistic effects could lead to the "U-shape" observed for the variation of the plateau modulus with the POXA concentration.

\section{Conclusions}

In the present work, Laponite/POXA nanocomposite hydrogels with various Laponite and POXA concentrations have been elaborated and investigated at both microscopic and macroscopic levels for the first time. The unique combination of rheology, NMR and SANS allowed to correlate the strong POXA adsorption onto the clay platelets to the original evolution of the elastic modulus of the hydrogels with the POXA concentration. Our results show that POXA adsorbs flat onto Laponite and the layers get denser as the polymer concentration increases. This adsorption is at the origin of two antagonistic effects on the mechanical properties of the colloidal system: screening of the electrostatic interactions and steric repulsion between the clay platelets, which contribute to decrease or increase (respectively) the elastic modulus of Laponite/POXA suspensions as the POXA concentration increases. These hydrogels are mainly electrostatic at low POXA concentration whereas they mainly correspond to steric hydrogels at saturation $\theta$ adsorption. Such results differ from the rheological behaviour usually reported for clay/polymer hydrogels in the literature[30,32,34,37], and particularly for 
the Laponite/PEO hydrogels with the same chain length $[\mathbf{3 7 , 4 2 , 4 7 ]}$. These latter indeed behave quite differently both at local and large length scales. Their affinity towards the clay layers is weaker, with not only trains, but also loop and tail conformations while the plateau moduli continuously decreases with the PEO concentration. It seems that the strong POXA adsorption promotes an original upturn in the interaction mechanism of the colloidal system, leading to strengthening of the nanocomposite hydrogels.

Further studies are underway in order to analyse the competitive adsorption between PEO and POXA and to determine whether the high affinity of POXA for Laponite layers could help to desorb PEO chains.

More generally, the U-shape dependence of the elastic modulus on the polymer concentration, here evidenced for nanocomposite Laponite/polymer hydrogels with a polymer (POXA) displaying a high affinity for the clay layers, is a very attracting and promising feature. It indeed opens the way towards a relatively simple way to tune the rheological behaviour of Laponitebased hydrogels. Along this line, we are currently investigating the effect of the post-addition of POXA chains on preformed Laponite/POXA hydrogels. Such kinds of gels could display a reinforcement of their elastic modulus upon the incorporation of additional polymer chains and could be, from this point of view, very interesting for tissue engineering applications. 


\section{Acknowledgements}

We would like to thank Laboratoire Léon Brillouin - Orphée reactor and FRM II which enabled us to perform the SANS experiments. This work is based upon experiments performed at the KWS-2 instrument operated by JCNS at the Heinz Maier-Leibnitz Zentrum (MLZ), Garching, Germany.

\section{References}

[1] K. Nagahama, D. Kawano, N. Oyama, A. Takemoto, T. Kumano, J. Kawakami, Self-Assembling Polymer Micelle/Clay Nanodisk/Doxorubicin Hybrid Injectable Gels for Safe and Efficient Focal Treatment of Cancer, Biomacromolecules. 16 (2015) 880-889. https://doi.org/10.1021/bm5017805.

[2] S. Wang, Y. Wu, R. Guo, Y. Huang, S. Wen, M. Shen, J. Wang, X. Shi, Laponite Nanodisks as an Efficient Platform for Doxorubicin Delivery to Cancer Cells, Langmuir. 29 (2013) 5030-5036. https://doi.org/10.1021/la4001363.

[3] N. Oyama, H. Minami, D. Kawano, M. Miyazaki, T. Maeda, K. Toma, A. Hotta, K. Nagahama, A nanocomposite approach to develop biodegradable thermogels exhibiting excellent cellcompatibility for injectable cell delivery, Biomater. Sci. 2 (2014) 1057-1062. https://doi.org/10.1039/C4BM00074A.

[4] B. Ruzicka, E. Zaccarelli, A fresh look at the Laponite phase diagram, Soft Matter. 7 (2011) 1268-1286. https://doi.org/10.1039/COSM00590H.

[5] E. Loizou, P. Butler, L. Porcar, G. Schmidt, Dynamic Responses in Nanocomposite Hydrogels, Macromolecules. 39 (2006) 1614-1619. https://doi.org/10.1021/ma0517547.

[6] P. Schexnailder, E. Loizou, L. Porcar, P. Butler, G. Schmidt, Heterogeneity in nanocomposite hydrogels from poly(ethylene oxide) cross-linked with silicate nanoparticles, Phys. Chem. Chem. Phys. 11 (2009) 2760-2766. https://doi.org/10.1039/B820452G.

[7] Q. Zhang, X. Li, Y. Zhao, L. Chen, Preparation and performance of nanocomposite hydrogels based on different clay, Appl. Clay Sci. 46 (2009) 346-350. https://doi.org/10.1016/j.clay.2009.09.003.

[8] K. Haraguchi, T. Takehisa, S. Fan, Effects of Clay Content on the Properties of Nanocomposite Hydrogels Composed of Poly(N-isopropylacrylamide) and Clay, Macromolecules. 35 (2002) 10162-10171. https://doi.org/10.1021/ma021301r.

[9] K. Haraguchi, R. Farnworth, A. Ohbayashi, T. Takehisa, Compositional Effects on Mechanical Properties of Nanocomposite Hydrogels Composed of Poly(N,N-dimethylacrylamide) and Clay, Macromolecules. 36 (2003) 5732-5741. https://doi.org/10.1021/ma034366i.

[10] W.-C. Lin, W. Fan, A. Marcellan, D. Hourdet, C. Creton, Large Strain and Fracture Properties of Poly(dimethylacrylamide)/Silica Hybrid Hydrogels, Macromolecules. 43 (2010) 2554-2563. https://doi.org/10.1021/ma901937r.

[11] S. Rose, A. Dizeux, T. Narita, D. Hourdet, A. Marcellan, Time Dependence of Dissipative and Recovery Processes in Nanohybrid Hydrogels, Macromolecules. 46 (2013) 4095-4104. https://doi.org/10.1021/ma400447j.

[12] K. Haraguchi, T. Takehisa, Nanocomposite Hydrogels: A Unique Organic-Inorganic Network Structure with Extraordinary Mechanical, Optical, and Swelling/De-swelling Properties, Adv. 
Mater. 14 (2002) 1120-1124. https://doi.org/10.1002/1521-4095(20020816)14:16<1120::AIDADMA1120>3.0.CO;2-9.

[13] K. Haraguchi, Nanocomposite hydrogels, Curr. Opin. Solid State Mater. Sci. 11 (2007) 47-54. https://doi.org/10.1016/j.cossms.2008.05.001.

[14] J. Lal, L. Auvray, Interaction of polymer with clays, J. Appl. Crystallogr. 33 (2000) 673-676. https://doi.org/10.1107/S0021889899013308.

[15] E. Loizou, P. Butler, L. Porcar, E. Kesselman, Y. Talmon, A. Dundigalla, G. Schmidt, Large Scale Structures in Nanocomposite Hydrogels, Macromolecules. 38 (2005) 2047-2049. https://doi.org/10.1021/ma047411a.

[16] A. Nelson, T. Cosgrove, A Small-Angle Neutron Scattering Study of Adsorbed Poly(ethylene oxide) on Laponite, Langmuir. 20 (2004) 2298-2304. https://doi.org/10.1021/la035268t.

[17] J.O. Fossum, J. Samseth, K. Mortensen, A Small-Angle-Neutron-Scattering Study of a Synthetic Clay, (1998) I38.73.

[18] S. Bhatia, J. Barker, A. Mourchid, Scattering of Disklike Particle Suspensions: Evidence for Repulsive Interactions and Large Length Scale Structure from Static Light Scattering and UltraSmall-Angle Neutron Scattering, Langmuir. 19 (2003) 532-535. https://doi.org/10.1021/la0265732.

[19] J. Lal, L. Auvray, Interaction of Polymer with Discotic Clay Particles, Mol. Cryst. Liq. Cryst. Sci. Technol. Sect. Mol. Cryst. Liq. Cryst. 356 (2001) 503-515. https://doi.org/10.1080/10587250108023729.

[20] K. Suman, Y.M. Joshi, Microstructure and Soft Glassy Dynamics of an Aqueous Laponite Dispersion, Langmuir. 34 (2018) 13079-13103. https://doi.org/10.1021/acs.langmuir.8b01830.

[21] M. Ghadiri, H. Hau, W. Chrzanowski, H. Agus, R. Rohanizadeh, Laponite clay as a carrier for in situ delivery of tetracycline, RSC Adv. 3 (2013) 20193-20201. https://doi.org/10.1039/C3RA43217C.

[22] J. Li, Y. Yang, Y. Yu, Q. Li, G. Tan, Y. Wang, W. Liu, W. Pan, LAPONITE ${ }^{\circledR}$ nanoplatform functionalized with histidine modified oligomeric hyaluronic acid as an effective vehicle for the anticancer drug methotrexate, J. Mater. Chem. B. 6 (2018) 5011-5020. https://doi.org/10.1039/C8TB01284A.

[23] H. Tomás, C.S. Alves, J. Rodrigues, Laponite ${ }^{\circledR}:$ A key nanoplatform for biomedical applications?, Nanomedicine Nanotechnol. Biol. Med. 14 (2018) 2407-2420. https://doi.org/10.1016/j.nano.2017.04.016.

[24] T.X. Phuoc, P. Wang, D. McIntyre, L. Shadle, Synthesis and characterization of a thixotropic coal-water slurry for use as a liquid fuel, Fuel Process. Technol. 127 (2014) 105-110. https://doi.org/10.1016/j.fuproc.2014.06.019.

[25] A. Mourchid, E. Lécolier, H. Van Damme, P. Levitz, On Viscoelastic, Birefringent, and Swelling Properties of Laponite Clay Suspensions: Revisited Phase Diagram, Langmuir. 14 (1998) 47184723. https://doi.org/10.1021/la980117p.

[26] P. Mongondry, J.F. Tassin, T. Nicolai, Revised state diagram of Laponite dispersions, J. Colloid Interface Sci. 283 (2005) 397-405. https://doi.org/10.1016/j.jcis.2004.09.043.

[27] A. Mourchid, A. Delville, J. Lambard, E. LeColier, P. Levitz, Phase Diagram of Colloidal Dispersions of Anisotropic Charged Particles: Equilibrium Properties, Structure, and Rheology of Laponite Suspensions, Langmuir. 11 (1995) 1942-1950. https://doi.org/10.1021/la00006a020.

[28] A.K. Atmuri, G.A. Peklaris, S. Kishore, S.R. Bhatia, A re-entrant glass transition in colloidal disks with adsorbing polymer, Soft Matter. 8 (2012) 8965-8971. https://doi.org/10.1039/c2sm25311a.

[29] R.P. Mohanty, Y.M. Joshi, Chemical stability phase diagram of aqueous Laponite dispersions, Appl. Clay Sci. 119 (2016) 243-248. https://doi.org/10.1016/j.clay.2015.10.021.

[30] A. Loiseau, J.-F. Tassin, Model Nanocomposites Based on Laponite and Poly(ethylene oxide): Preparation and Rheology, Macromolecules. 39 (2006) 9185-9191. https://doi.org/10.1021/ma061324w. 
[31] G. Schmidt, A.I. Nakatani, P.D. Butler, C.C. Han, Small-Angle Neutron Scattering from Viscoelastic Polymer-Clay Solutions, Macromolecules. 35 (2002) 4725-4732. https://doi.org/10.1021/ma0115141.

[32] J. Zebrowski, V. Prasad, W. Zhang, L.M. Walker, D.A. Weitz, Shake-gels: shear-induced gelation of laponite-PEO mixtures, Colloids Surf. Physicochem. Eng. Asp. 213 (2003) 189-197. https://doi.org/10.1016/S0927-7757(02)00512-5.

[33] R. De Lisi, M. Gradzielski, G. Lazzara, S. Milioto, N. Muratore, S. Prévost, Aqueous Laponite Clay Dispersions in the Presence of Poly(ethylene oxide) or Poly(propylene oxide) Oligomers and their Triblock Copolymers, J. Phys. Chem. B. 112 (2008) 9328-9336. https://doi.org/10.1021/jp8024073.

[34] D.C. Pozzo, L.M. Walker, Reversible shear gelation of polymer-clay dispersions, Colloids Surf. Physicochem. Eng. Asp. 240 (2004) 187-198. https://doi.org/10.1016/j.colsurfa.2004.04.040.

[35] S. Morariu, M. Bercea, L.M. Gradinaru, I. Rosca, M. Avadanei, Versatile poly(vinyl alcohol)/clay physical hydrogels with tailorable structure as potential candidates for wound healing applications, Mater. Sci. Eng. C. 109 (2020) 110395. https://doi.org/10.1016/j.msec.2019.110395.

[36] W. Sun, Y. Yang, T. Wang, X. Liu, C. Wang, Z. Tong, Large amplitude oscillatory shear rheology for nonlinear viscoelasticity in hectorite suspensions containing poly(ethylene glycol), Polymer. 52 (2011) 1402-1409. https://doi.org/10.1016/j.polymer.2011.01.048.

[37] C. Lorthioir, M. Khalil, V. Wintgens, C. Amiel, Segmental Motions of Poly(ethylene glycol) Chains Adsorbed on Laponite Platelets in Clay-Based Hydrogels: A NMR Investigation, Langmuir. 28 (2012) 7859-7871. https://doi.org/10.1021/la3010757.

[38] F. Ordikhani, M. Dehghani, A. Simchi, Antibiotic-loaded chitosan-Laponite films for local drug delivery by titanium implants: cell proliferation and drug release studies, J. Mater. Sci. Mater. Med. 26 (2015) 269. https://doi.org/10.1007/s10856-015-5606-0.

[39] C.-W. Chang, A. van Spreeuwel, C. Zhang, S. Varghese, PEG/clay nanocomposite hydrogel: a mechanically robust tissue engineering scaffold, Soft Matter. 6 (2010) 5157-5164. https://doi.org/10.1039/COSM00067A.

[40] S. Kishore, S. Srivastava, S.R. Bhatia, Microstructure of colloid-polymer mixtures containing charged colloidal disks and weakly-adsorbing polymers, Polymer. 105 (2016) 461-471. https://doi.org/10.1016/j.polymer.2016.07.091.

[41] A. Nelson, T. Cosgrove, Dynamic light scattering studies of poly(ethylene oxide) adsorbed on Laponite: layer conformation and its effect on particle stability, Langmuir ACS J. Surf. Colloids. 20 (2004) 10382-10388. https://doi.org/10.1021/la049323p.

[42] P. Mongondry, Structure et comportement rhéologique des suspensions aqueuses de Laponite en présence de plusieurs additifs, thesis, Le Mans, 2003. http://www.theses.fr/2003LEMA1001 (accessed February 16, 2020).

[43] L. Zulian, B. Ruzicka, G. Ruocco, Influence of an adsorbing polymer on the aging dynamics of Laponite clay suspensions, Philos. Mag. 88 (2008) 4213-4221. https://doi.org/10.1080/14786430802581876.

[44] H.A. Baghdadi, H. Sardinha, S.R. Bhatia, Rheology and gelation kinetics in laponite dispersions containing poly(ethylene oxide), J. Polym. Sci. Part B Polym. Phys. 43 (2005) 233-240. https://doi.org/10.1002/polb.20317.

[45] A. Fall, D. Bonn, Shear thickening of Laponite suspensions with poly(ethylene oxide), Soft Matter. 8 (2012) 4645-4651. https://doi.org/10.1039/C2SM07089H.

[46] V. Can, O. Okay, Shake gels based on Laponite-PEO mixtures: effect of polymer molecular weight, Des. Monomers Polym. 8 (2005) 453-462. https://doi.org/10.1163/1568555054937917.

[47] S. Rossi, P.F. Luckham, T.F. Tadros, Influence of non-ionic polymers on the rheological behaviour of $\mathrm{Na}+$-montmorillonite clay suspensions. Part II. Homopolymer ethyleneoxide and polypropylene oxide-polyethylene oxide ABA copolymers, Colloids Surf. Physicochem. Eng. Asp. 215 (2003) 1-10. https://doi.org/10.1016/S0927-7757(02)00446-6. 
[48] X. Frielinghaus, M. Brodeck, O. Holderer, H. Frielinghaus, Confined Polymer Dynamics on Clay Platelets, Langmuir. 26 (2010) 17444-17448. https://doi.org/10.1021/la102667k.

[49] R. Luxenhofer, Y. Han, A. Schulz, J. Tong, Z. He, A.V. Kabanov, R. Jordan, Poly(2-oxazoline)s as Polymer Therapeutics, Macromol. Rapid Commun. 33 (2012) 1613-1631. https://doi.org/10.1002/marc.201200354.

[50] R. Luxenhofer, Y. Han, A. Schulz, J. Tong, Z. He, A.V. Kabanov, R. Jordan, Poly(2-oxazoline)s as Polymer Therapeutics, Macromol. Rapid Commun. 33 (2012) 1613-1631. https://doi.org/10.1002/marc.201200354.

[51] G. Volet, V. Chanthavong, V. Wintgens, C. Amiel, Synthesis of Monoalkyl End-Capped Poly(2methyl-2-oxazoline) and Its Micelle Formation in Aqueous Solution, Macromolecules. 38 (2005) 5190-5197. https://doi.org/10.1021/ma050407u.

[52] A. Radulescu, V. Pipich, H. Frielinghaus, M.-S. Appavou, KWS-2, the high intensity / wideQ-range small-angle neutron diffractometer for soft-matter and biology at FRM II, J. Phys. Conf. Ser. 351 (2012) 012026. https://doi.org/10.1088/1742-6596/351/1/012026.

[53] G. Le Fer, C. Le Cœur, J.-M. Guigner, C. Amiel, G. Volet, Biocompatible Soft Nanoparticles with Multiple Morphologies Obtained from Nanoprecipitation of Amphiphilic Graft Copolymers in a Backbone-Selective Solvent, Langmuir. 33 (2017) 2849-2860. https://doi.org/10.1021/acs.langmuir.7b00471.

[54] SasView/sasview, GitHub. (2020). https://github.com/SasView/sasview (accessed February 16, 2020).

[55] C. Flood, T. Cosgrove, Y. Espidel, E. Welfare, I. Howell, P. Revell, Fourier-Transform Carr-Purcell-Meiboom-Gill NMR Experiments on Polymers in Colloidal Dispersions: How Many Polymer Molecules per Particle?, Langmuir. 24 (2008) 7875-7880. https://doi.org/10.1021/la800144c.

[56] J. Gregory, Polymers at interfaces, by G. J. Fleer, M. A. Cohen Stuart, J. M. H. M. Scheutjens, T. Cosgrove and B. Vincent. Chapman and Hall, London, 1993. Pp. xv + 502, price f65.00. ISBN 0412-58160-4, Polym. Int. 36 (1995) 102-102. https://doi.org/10.1002/pi.1995.210360115.

[57] Y.-H. Shen, Estimation of surface area of montmorillonite by ethylene oxide chain adsorption, Chemosphere. 48 (2002) 1075-1079. https://doi.org/10.1016/S0045-6535(02)00200-X.

[58] G. Volet, L. Auvray, C. Amiel, Monoalkyl Poly(2-methyl-2-oxazoline) Micelles. A Small-Angle Neutron Scattering Study, J. Phys. Chem. B. 113 (2009) 13536-13544. https://doi.org/10.1021/jp9029634.

[59] A. Guinier, G. Fournet, C.B. Walker, K.L. Yudowitch, Small-Angle Scattering of X-Rays., Wiley, 1955.

[60] T. Nicolai, S. Cocard, Light Scattering Study of the Dispersion of Laponite, Langmuir. 16 (2000) 8189-8193. https://doi.org/10.1021/la9915623. 


\title{
Supporting Material
}

\section{Laponite/poly(2-methyl-2-oxazoline) hydrogels : interplay}

\section{between local structure and rheological behaviour}

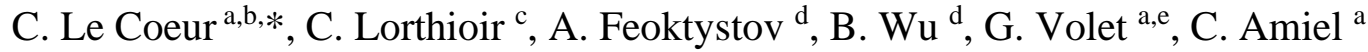

a Université Paris Est Créteil, CNRS, Institut Chimie et Matériaux Paris Est, 2 rue Henri Dunant, 94320 Thiais, France

b Laboratoire Léon Brillouin, CEA-CNRS (UMR-12), CEA Saclay, Université Paris-Saclay, 91191 Gif-sur-Yvette Cedex, France

c Sorbonne Université, CNRS, Collège de France, Laboratoire de Chimie de la Matière Condensée de Paris, 4 Place Jussieu, 75005 Paris, France

d Heinz Maier Leibnitz Zentrum, Jülich Centre for Neutron Science, Lichtenbergstraße 1, 85748 Garching, Germany

e Université d'Evry Val d'Essonne, Rue du Père Jarlan, 91025 Evry Cedex, France 


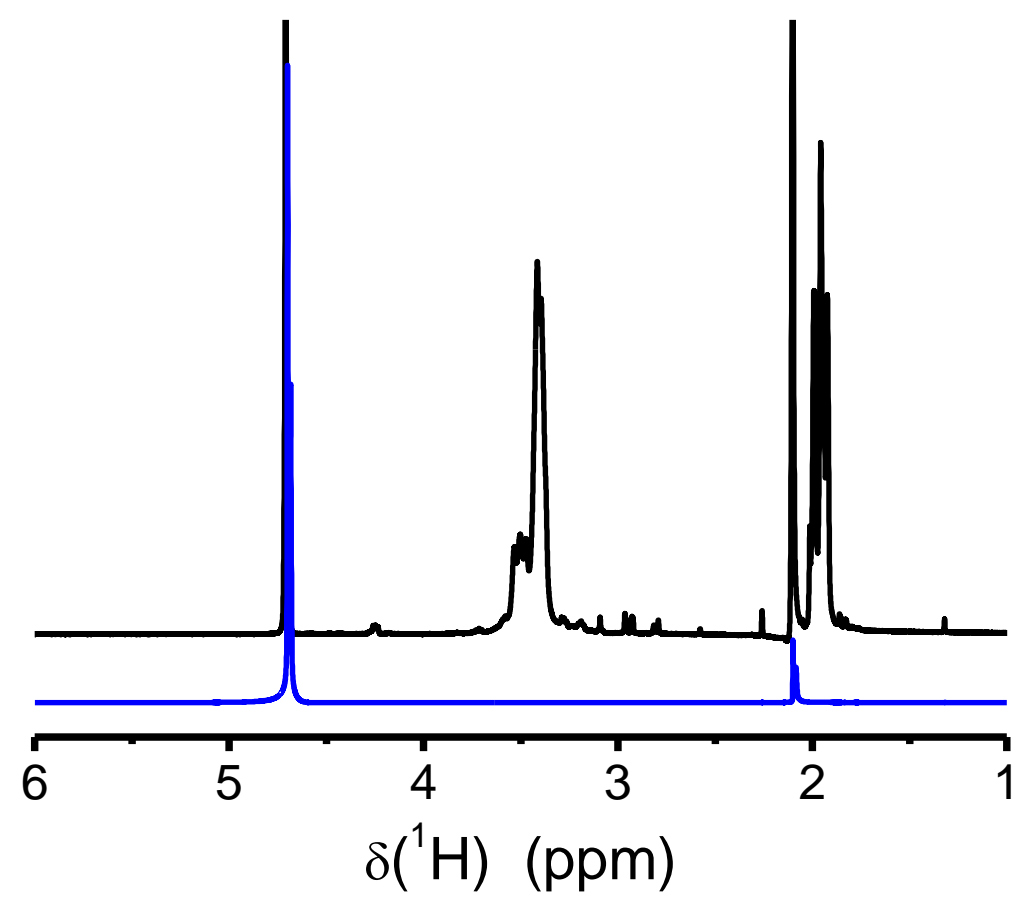

Figure S1. ${ }^{1} \mathrm{H}$ NMR spectra $(400 \mathrm{MHz})$ of a POXA solution in $\mathrm{D}_{2} \mathrm{O}\left(\mathrm{c}_{\mathrm{p}}=0.66 \mathrm{wt} \%, \mathrm{c}_{\text {lap }}=0 \mathrm{wt} \%\right.$; black line $)$ and a Laponite/POXA system $\left(\mathrm{c}_{\mathrm{p}}=0.66 \mathrm{wt} \%, \mathrm{c}_{\text {lap }}=2 \mathrm{wt} \%\right.$; blue line $)$, just after mixing both clay and polymer components in $\mathrm{D}_{2} \mathrm{O}$. 
(a)

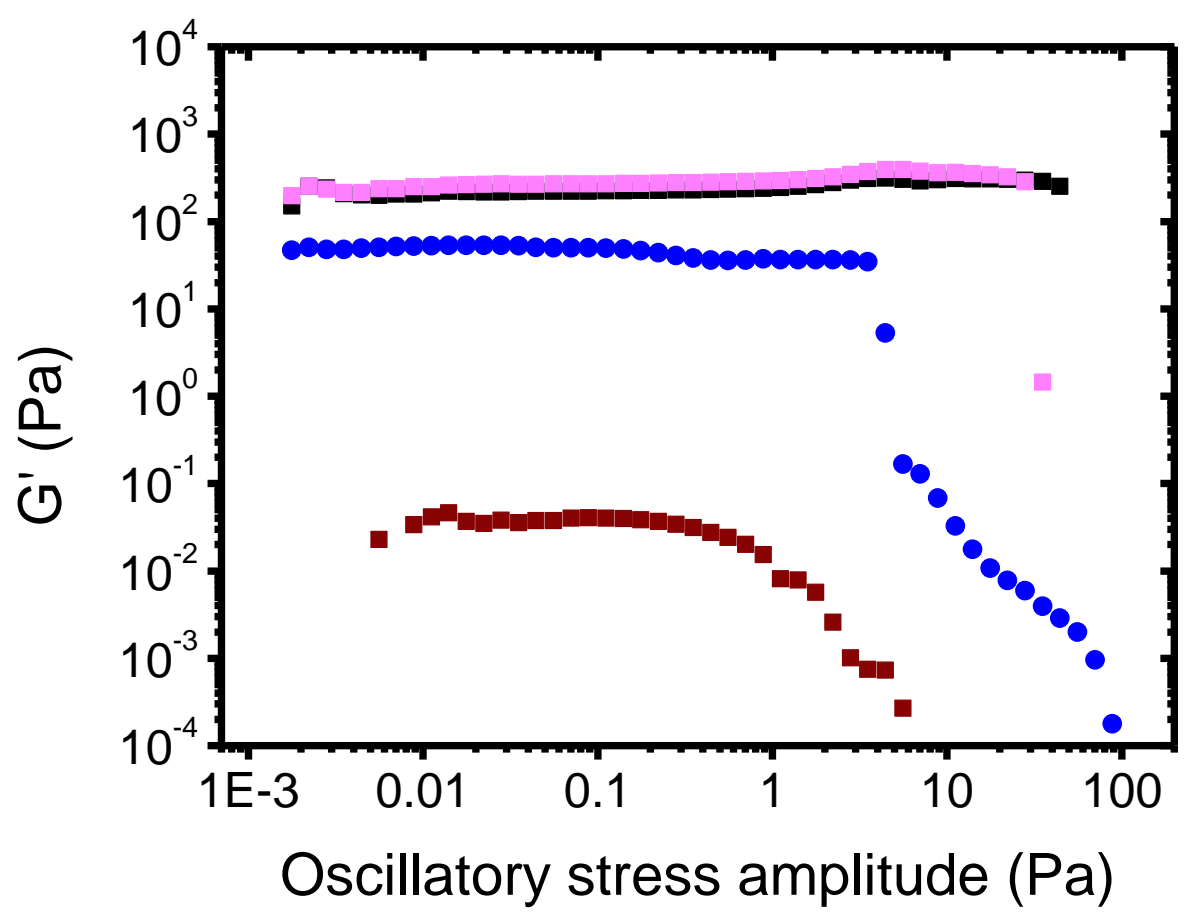

(b)

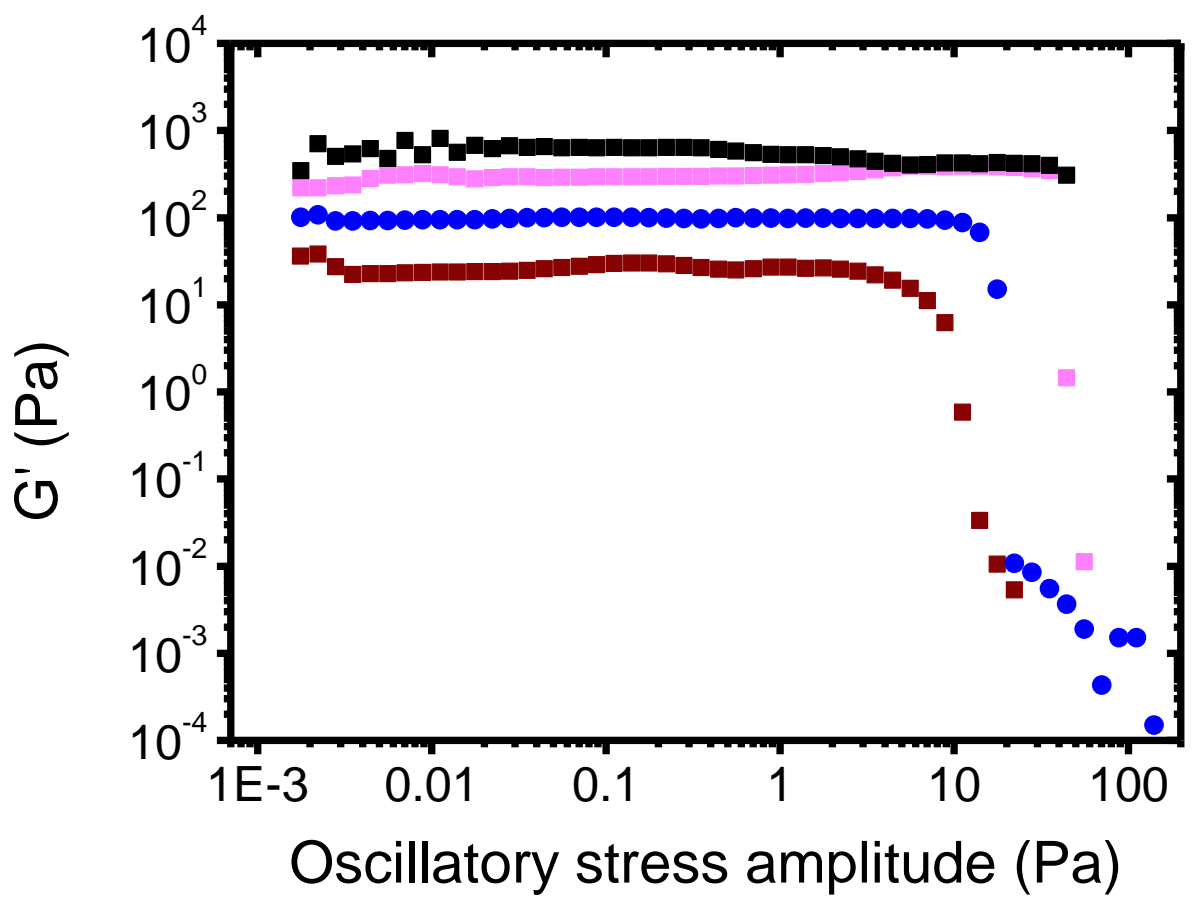

Figure S2. Storage shear moduli $\mathrm{G}^{\prime}$ as a function of the oscillatory stress amplitude for different Laponite/POXA hydrogels for various delays following the sample preparation: (a) 1 day, (b) 11 days. All the measurements were performed at $1 \mathrm{~Hz}$. The Laponite 
weight percent has been fixed to $2 \mathrm{wt} \%$ while the POXA concentration has been varied: $0.21(\square), 0.42(\square), 0.63(\bullet)$ and $1.89(\square)$ wt $\%$.

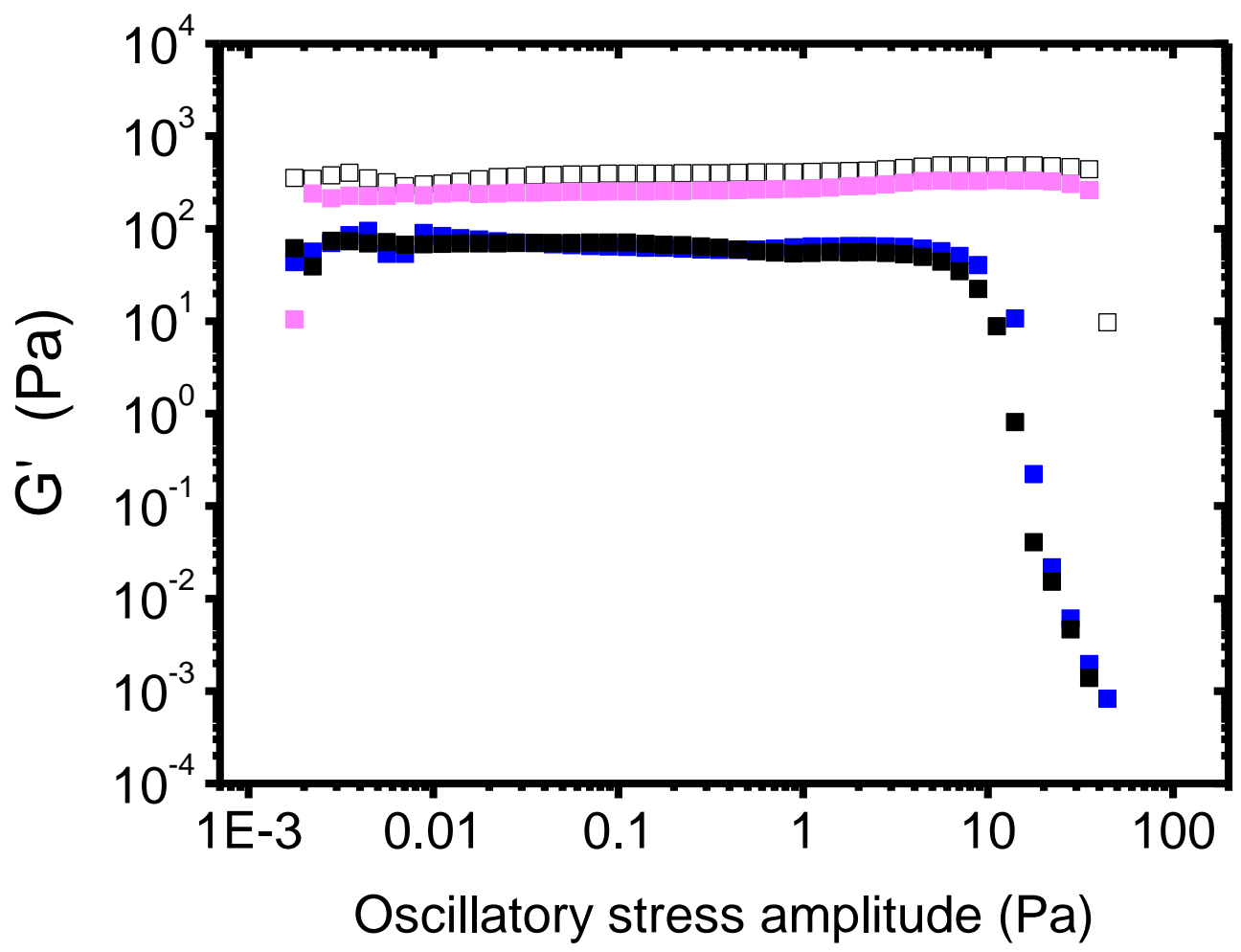

Figure S3. Evolution of the storage shear moduli $\mathrm{G}^{\prime}$ as a function of the oscillatory stress amplitude for Laponite/PEO hydrogels prepared with different values of the PEO concentration: $\mathrm{C}_{\mathrm{PEO}}$ $=0(\square), 0.21(\square), 0.66(\bullet)$ and $1.89(\square)$ wt $\%$. Such measurements were performed for a fixed Laponite concentration of $2 \mathrm{wt} \%$ and following 3 days after the sample preparation. It is worth noting that the degree of polymerization of the PEO chains used to prepare these hydrogels was similar to the one of the POXA chains considered in this work. 


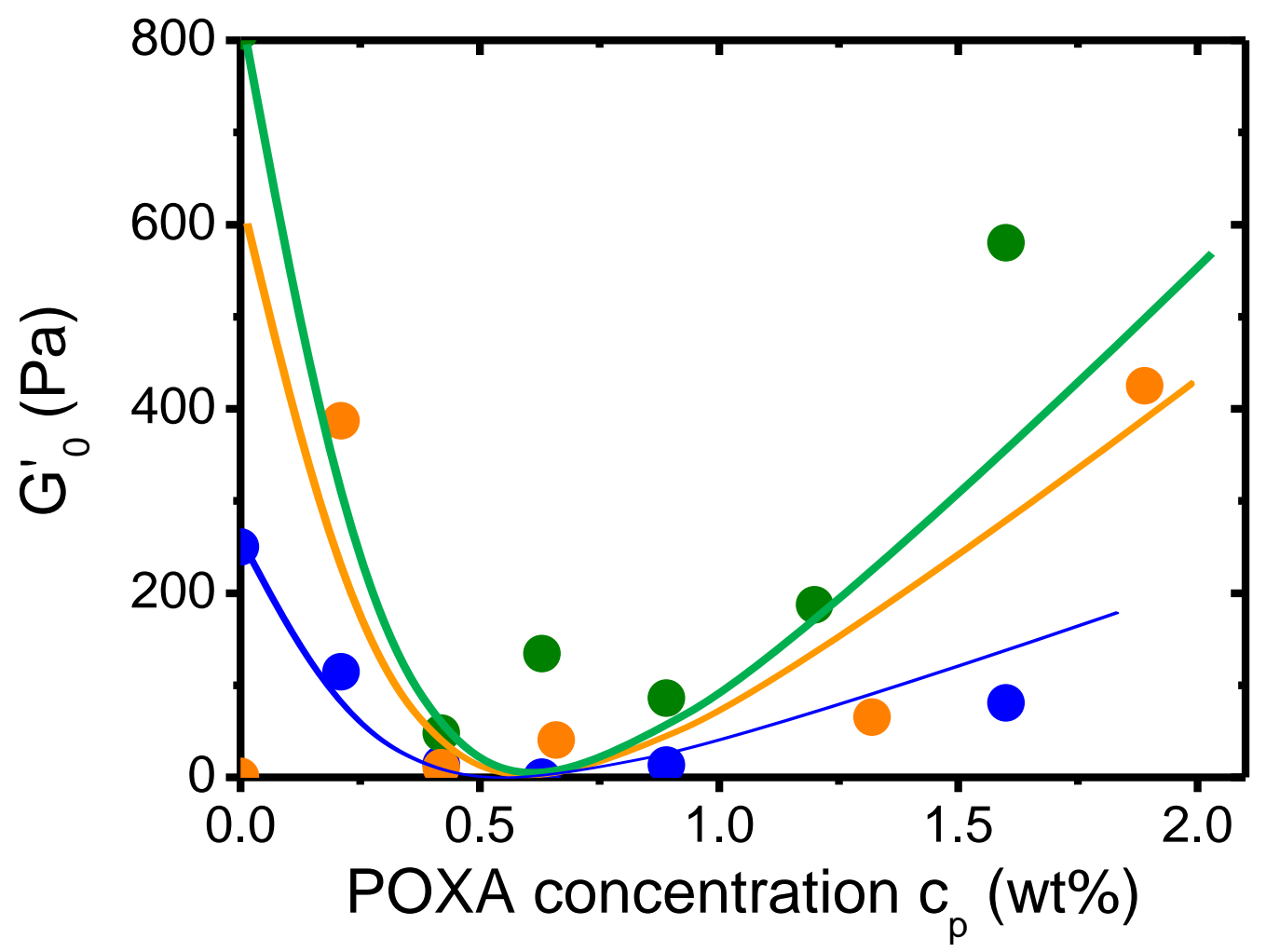

Figure S4. Evolution of the plateau modulus $\mathrm{G}_{0}{ }^{\prime}$ as a function of the POXA concentration for Laponite/POXA hydrogels, 3 days following their preparation. Such a variation was determined for a fixed Laponite concentration of $1.5(\bullet), 2.0(\bullet)$ and $2.5(\bullet) \mathrm{wt} \%$. The "U-shape" curves serve as guides for the eyes. 


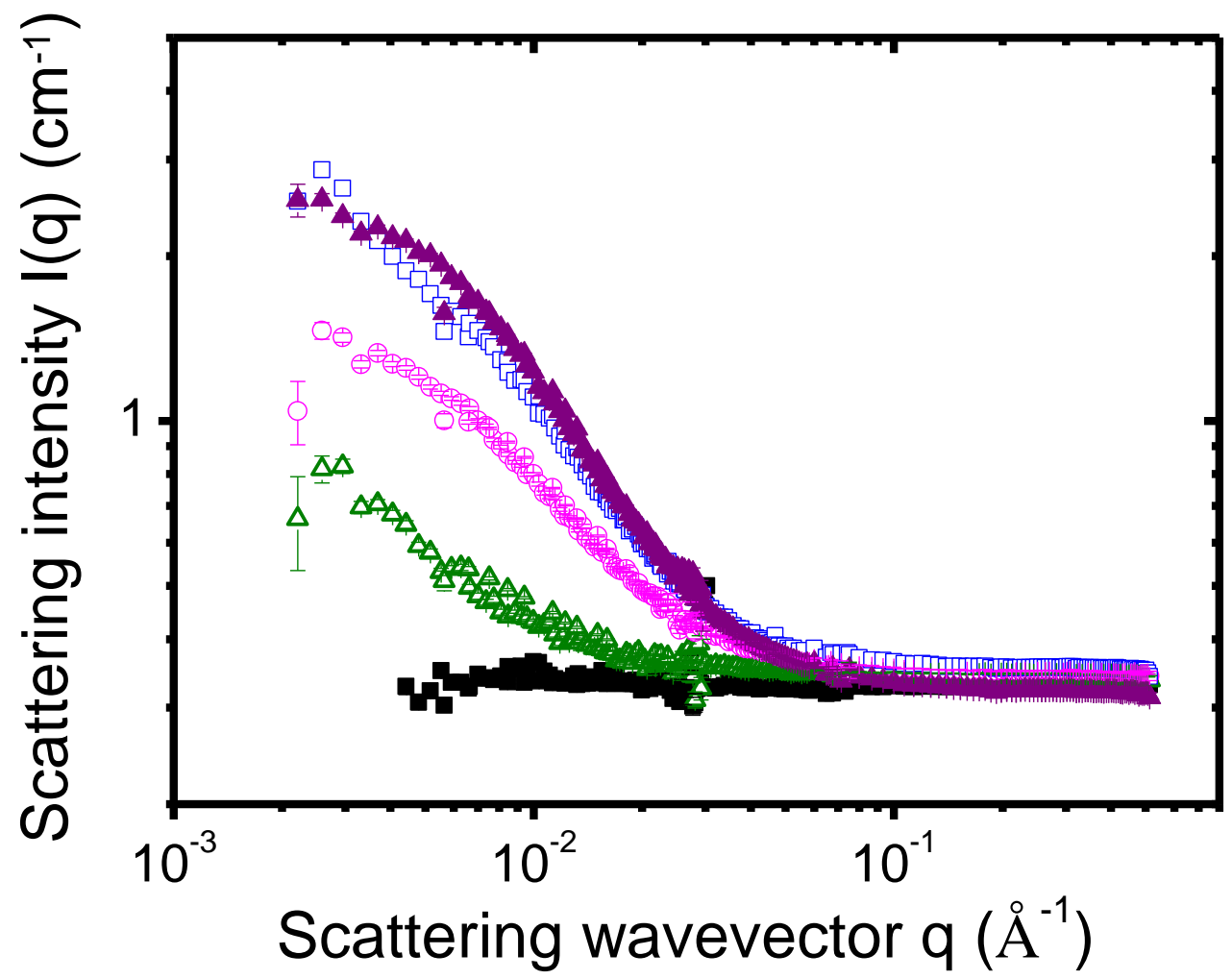

Figure S5. Scattered intensity $\mathrm{I}(\mathrm{q})$ of POXA as a function of the wavevector $\mathrm{q}$ for Laponite/POXA samples with different values of $c_{p}: c_{p}=0(\square), 0.42(\triangle), 0.63$ $(\bigcirc), 0.89(\square)$ and $1.20(\Delta)$ wt $\%$. The Laponite weight percentage is fixed at 2 wt\%. The proportion of $\mathrm{D}_{2} \mathrm{O}$ in the $\mathrm{H}_{2} \mathrm{O} / \mathrm{D}_{2} \mathrm{O}$ mixture is set to $68 \mathrm{wt} \%$ which corresponds to the contrast-matching conditions of the Laponite layers. Therefore, under these conditions, the scattered intensity only results from POXA. 


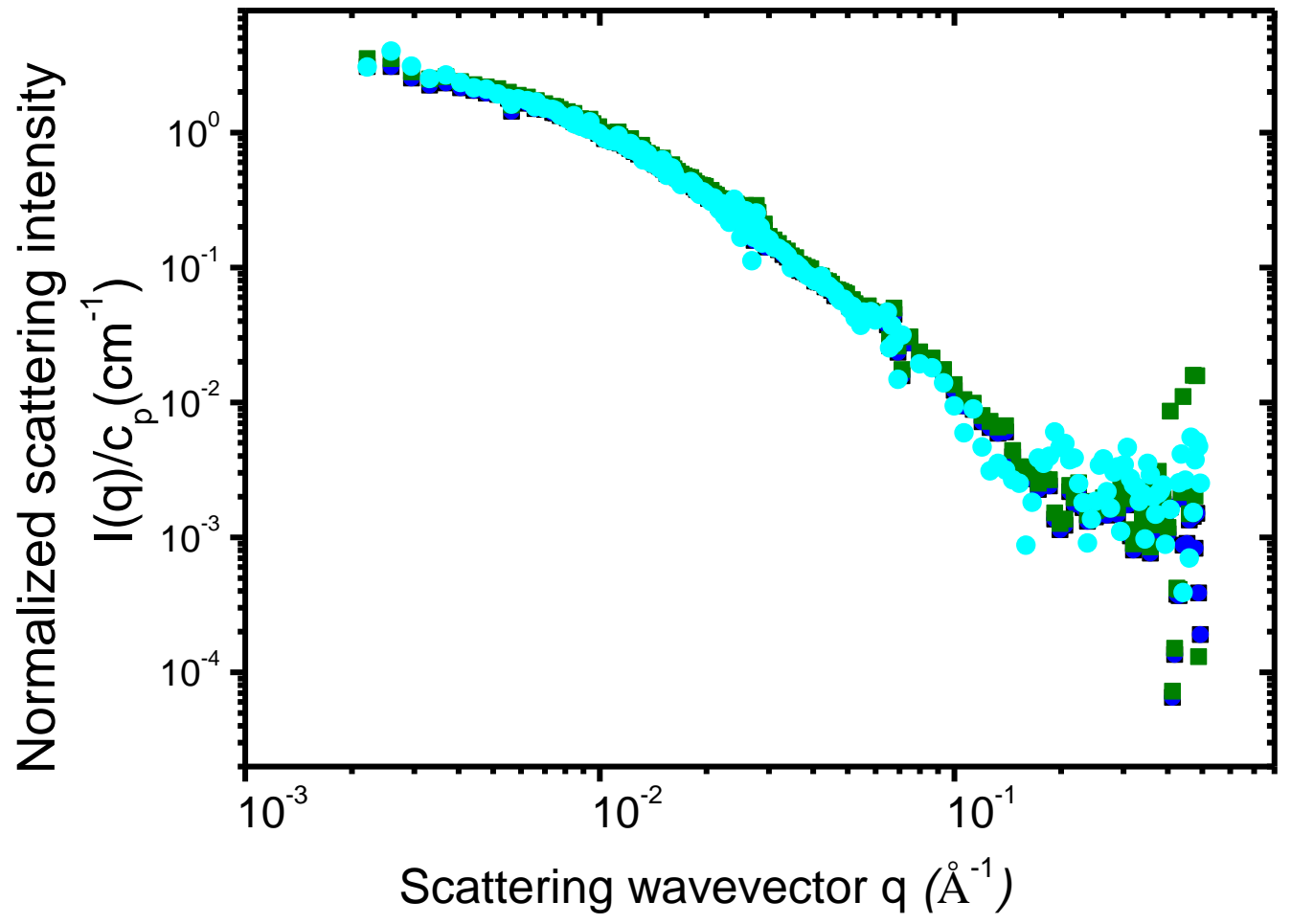

Figure S6. Normalized scattered intensity $\mathrm{I}(\mathrm{q}) / \mathrm{c}_{\mathrm{p}}$ of a Laponite/POXA sample with a Laponite weight percentage of $2 \mathrm{wt} \%$ and a POXA concentration $\mathrm{c}_{\mathrm{p}}$ of $0.66 \mathrm{wt} \%$, recorded using contrast-matching of the Laponite layers $\left(68 \mathrm{wt} \%\right.$ of $\mathrm{D}_{2} \mathrm{O}$ in the solvent mixture $\mathrm{H}_{2} \mathrm{O} / \mathrm{D}_{2} \mathrm{O}$ ). Measurements were performed after various delays following

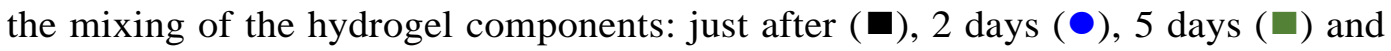
15 days $(\odot)$. 


\begin{tabular}{|c|c|c|c|c|c|c|c|}
\hline$c_{p}(\mathrm{wt} \%)$ & $R_{p}(\AA)$ & $h_{p}(\AA)$ & $D-h_{p}(\AA)$ & $\sigma_{D}(\AA)$ & $I_{0} / c_{p}\left(\mathrm{~cm}^{-1}\right)$ & $\begin{array}{c}M_{a} \\
\left(g . m o l^{-1}\right)\end{array}$ & $N_{c}$ \\
\hline 0.42 & 149 & 5 & 9 & 0.1 & 2 & 952000 & 280 \\
\hline 0.63 & 149 & 5 & 9 & 0.1 & 2 & 952000 & 280 \\
\hline 0.89 & 149 & 5 & 9 & 0.1 & 2 & 952000 & 280 \\
\hline 1.20 & 149 & 5 & 9 & 0.1 & 2 & 952000 & 280 \\
\hline
\end{tabular}

Table S1. Results obtained by fitting the SANS data using a model of polymer discs adsorbed on each layer of a clay platelet. The concentration of clay is constant and equal to $2 \mathrm{wt} \%$.

\begin{tabular}{|c|c|c|c|c|c|c|c|}
\hline$c_{p}(\mathrm{wt} \%)$ & $R_{p}(\AA)$ & $h_{p}(\AA)$ & $D-h_{p}(\AA)$ & $\sigma_{D}(\AA)$ & $I_{0} / c_{p}\left(\mathrm{~cm}^{-1}\right)$ & $\begin{array}{c}M_{a} \\
\left(g . m o l^{-1}\right)\end{array}$ & $N_{c}$ \\
\hline 0.42 & 149 & 5 & 9 & 0.1 & 0.4 & 185000 & 55 \\
\hline 0.63 & 149 & 5 & 9 & 0.1 & 0.85 & 420000 & 120 \\
\hline 0.89 & 149 & 5 & 9 & 0.1 & 1.85 & 880000 & 260 \\
\hline 1.20 & 149 & 5 & 9 & 0.1 & 1.9 & 900000 & 265 \\
\hline 1.80 & 149 & 5 & 9 & 0.1 & 2 & 952000 & 280 \\
\hline
\end{tabular}

Table S2. Results obtained by fitting the SANS data using a model of polymer discs adsorbed on each layer of a clay platelet. The concentration of clay is constant and equal to $2.5 \mathrm{wt} \%$. 